ARTICLE

\title{
A second generation leishmanization vaccine with a markerless attenuated Leishmania major strain using CRISPR gene editing
}

Wen-Wei Zhang (1) 1,8, Subir Karmakar2,8, Sreenivas Gannavaram²,8, Ranadhir Dey 2,8, Patrick Lypaczewski (iD 1, Nevien Ismail ${ }^{2}$, Abid Siddiqui ${ }^{2}$, Vahan Simonyan (1) ${ }^{2}$, Fabiano Oliveira (1) ${ }^{3}$, Iliano V. Coutinho-Abreu (1) ${ }^{3}$, Thiago DeSouza-Vieira ${ }^{3}$, Claudio Meneses ${ }^{3}$, James Oristian ${ }^{3}$, Tiago D. Serafim³ ${ }^{3}$ Abu Musa (i) ${ }^{4}$, Risa Nakamura4, Noushin Saljoughian ${ }^{5}$, Greta Volpedo ${ }^{5}$, Monika Satoskar ${ }^{2,7}$, Sanika Satoskar ${ }^{2,7}$, Pradeep K. Dagur ${ }^{6}$, J. Philip $\mathrm{McCoy}^{6}$, Shaden Kamhawi ${ }^{3}{ }^{3}$, Jesus G. Valenzuela (i] ${ }^{3}$, Shinjiro Hamano ${ }^{4}$, Abhay R. Satoskar (i) ${ }^{5 凶}$, Greg Matlashewski (iD) ${ }^{1 凶} \&$ Hira L. Nakhasi (i) ${ }^{2 凶}$

Leishmaniasis is a neglected tropical disease caused by Leishmania protozoa transmitted by infected sand flies. Vaccination through leishmanization with live Leishmania major has been used successfully but is no longer practiced because it resulted in occasional skin lesions. A second generation leishmanization is described here using a CRISPR genome edited L. major strain ( $\mathrm{LmCen}^{-/-}$). Notably, $\mathrm{LmCen}{ }^{-/-}$is a genetically engineered centrin gene knock-out mutant strain that is antibiotic resistant marker free and does not have detectable off-target mutations. Mice immunized with $L m \mathrm{Len}^{-/-}$have no visible lesions following challenge with $L$. major-infected sand flies, while non-immunized animals develop large and progressive lesions with a 2-log fold higher parasite burden. $\mathrm{LmCen}{ }^{-1-}$ immunization results in protection and an immune response comparable to leishmanization. $\mathrm{LmCen}^{-/-}$is safe since it is unable to cause disease in immunocompromised mice, induces robust host protection against vector sand fly challenge and because it is marker free, can be advanced to human vaccine trials.

\footnotetext{
${ }^{1}$ Department of Microbiology and Immunology, McGill University, Montreal, QC H3A 2B4, Canada. ${ }^{2}$ Division of Emerging and Transfusion Transmitted Diseases, CBER, FDA, Silver Spring, MD 20993, USA. ${ }^{3}$ Vector Molecular Biology Section, Laboratory of Malaria and Vector Research, National Institute of Allergy and Infectious Diseases, National Institutes of Health, Rockville, MD 20852, USA. ${ }^{4}$ Department of Parasitology, Institute of Tropical Medicine (NEKKEN), The Joint Usage/Research Center on Tropical Disease, Nagasaki University, Nagasaki, Japan and Nagasaki University Graduate School of Biomedical Sciences Doctoral Leadership Program, Nagasaki, Japan. ${ }^{5}$ Department of Pathology and Microbiology, Ohio State University, Columbus, OH 43210, USA. ${ }^{6}$ National Institute of Heart, Lung and Blood Institute, NIH, Bethesda, MD 20852, USA. ${ }^{7}$ Present address: Northeast Ohio Medical University, Rootstown, Ohio 44272, USA. ${ }^{8}$ These authors contributed equally: Wen-Wei Zhang, Subir Karmakar, Sreenivas Gannavaram, Ranadhir Dey.

凶email: Abhay.Satoskar@osumc.edu; greg.matlashewski@mcgill.ca; Hira.Nakhasi@fda.hhs.gov
} 
eishmaniasis is a neglected disease caused by infection with protozoans of the genus Leishmania that is transmitted by infected sand flies ${ }^{1}$. Worldwide, an estimated 1 billion people are at risk of infection in tropical and subtropical countries where up to 1.7 million new cases in 98 countries occur each $y^{2} \mathrm{r}^{2,3}$. The disease pathology ranges from localized skin ulcers (cutaneous leishmaniasis, CL) to fatal systemic disease (visceral leishmaniasis, VL), depending on the species of the infecting Leishmania parasite ${ }^{1,4}$. Treatment options for both VL and CL are limited and there is poor surveillance in the most highly endemic countries ${ }^{1,5}$. A prophylactic vaccine would be an effective intervention for protection against this disease, reducing transmission and supporting the elimination of leishmaniasis globally. Currently there are no available vaccines against any form of human leishmaniasis.

Unlike most parasitic infections, patients who recover from leishmaniasis naturally or following drug treatment develop immunity against reinfection indicating that the development of an effective vaccine should be feasible ${ }^{6-8}$. Furthermore, leishmanization, a process in which deliberate infections with a low dose of virulent Leishmania major provides greater than $90 \%$ protection against reinfection and has been used in several countries of the Middle East and the former Soviet Union ${ }^{9-11}$. Leishmanization is however no longer practiced due to safety concerns regarding skin lesions that last for months at the site of inoculation. The overall strategy of this study is to develop the next generation leishmanization that is safer by providing a protective immune response against cutaneous leishmaniasis without causing skin lesions.

In case of leishmaniasis cell-mediated immunity is critical, and particularly, CD4 $\mathrm{T}$ cells play a crucial role in the protection against $\mathrm{CL}^{12}$. Specifically, host defense involves Th1 response due to $\mathrm{T}$ - cells primed by antigen-presenting cells producing IL-12 ${ }^{13}$. Production of IL-12 by antigen-presenting cells and IFN $\gamma$ by $\mathrm{T}$ cells are crucial for controlling the parasite numbers ${ }^{13}$. In contrast, Th2 cytokines, mainly IL-4, IL-5 and IL-13, antiinflammatory cytokines, suppress host immunity and help parasite survival while minimizing the tissue damage due to unchecked inflammation ${ }^{13,14}$. The differential effects of Th1 and Th2 dichotomy in cutaneous leishmaniasis is extensively studied in murine models ${ }^{15}$.

Studies with several candidate vaccines against CL including leishmanization have demonstrated that the establishment of predominant Th1 type of immune response correlated with protection $^{16-18}$. In murine leishmanization models, it is well established that IFN- $\gamma$ producing CD4 Th1 cells are essential in mediating protective immunity against re-infection ${ }^{19,20}$. Multifunctional effector Th1 cells which also produce high IFN- $\gamma$ play a crucial role in host protection ${ }^{21}$. Recently it has been shown in leishmanized mice that rapidly recruited short-lived effector $\mathrm{T}$ cells producing IFN- $\gamma$ confer significant level of protection and could be used as a biomarker of host protection ${ }^{22,23}$. These studies collectively show that any effective vaccine should similarly maintain these antigen specific CD4 $\mathrm{T}$ cell populations long enough to induce a robust protection against reinfection.

Centrin is a calcium-binding protein and essential in the duplication of centrosomes in eukaryotes including Leishma$n_{i}{ }^{24,25}$. Previously, we have shown that centrin gene-deficient Leishmania donovani parasites are viable in axenic promastigote culture but do not proliferate in infected macrophages and are highly efficacious as a live vaccine in animal models ${ }^{26-31}$. However, using live-attenuated $L$. donovani as a vaccine in humans is high-risk because of the potential for visceralization resulting in fatal visceral disease. Further, previously generated gene deleted L. donovani strains required the incorporation of antibiotic resistance marker genes. The presence of antibiotic resistance genes in any attenuated live vaccine renders the vaccine unacceptable by regulatory agencies for human vaccine trials.

To overcome these drawbacks, we used CRISPR-Cas genome editing recently established for Leishmania ${ }^{32-34}$ to generate an attenuated $L$. major centrin gene deletion mutant $\left(\mathrm{LmCen}^{-/-}\right)$. This represents a major milestone because $\mathrm{LmCen}^{-1-}$ contains no antibiotic-resistant selection genes, an essential prerequisite for approval by regulatory agencies and advancement to human trials. L. major was used because this species is safer than $L$. donovani since $L$. major remains in the skin at the site of infection and does not cause visceral disease ${ }^{1,4}$. As demonstrated within, vaccination with $\mathrm{LmCen}^{-1-}$ is safe, immunogenic and protective against sand fly transmitted $L$. major infection, that mimics natural infection in highly relevant cutaneous leishmaniasis animal models meeting efficacy and ethical standards for advancement to human clinical studies.

\section{Results}

Generation of centrin deficient $\mathrm{LmCen}^{-/-}$by CRISPR-Cas. CRISPR-Cas genome editing has recently been developed to delete Leishmania genes with or without integration of antibiotic selection markers into the genome $\mathrm{e}^{32-34}$. The experimental approach used to delete the centrin gene (Gene ID: LmjF.22.1410) from L. major is detailed in Fig. 1a-d. Two guide sequences targeted to the $5^{\prime}$ and $3^{\prime}$ flanking sequences of the centrin gene were designed and cloned into the Leishmania CRISPR vector pLdCN $^{33,34}$ (Fig. 1a) and transfected into L. major (Friedlin V9) promastigotes. To delete the centrin gene sequence precisely at the locations determined by the 2 guide RNA sequences flanking the centrin gene without using marker gene replacement, a 50nucleotide oligonucleotide donor DNA sequence was transfected into the promastigotes containing the CRISPR expression vector pLdCNa\&b as previously described ${ }^{33}$. The donor DNA consisted of 25 nucleotides $5^{\prime}$ from the upstream gRNAa cleavage site and 25 nucleotides $3^{\prime}$ from the downstream gRNAb cleavage site (Fig. 1b). The exact targeted sequences flanking the centrin gene and diagnostic PCR primers are shown in Supplementary Fig. 1A.

L. donovani centrin null promastigotes proliferate slower than wildtype promastigotes ${ }^{35}$. Since centrin-null promastigotes were selection marker free, this slower proliferation phenotype was used to identify centrin null $L$. major promastigotes. The CRISPR-genome edited L. major promastigotes were subjected to single-cell cloning in 96 well plates; the relatively slow-growing clones were identified, expanded and subjected to PCR analysis with the primers flanking the centrin gene as shown in Fig. 1b. An example of a PCR analysis of a slow-growing clone with the loss of the centrin gene is shown in Fig. 1c. Sequence analysis of the 604 bp PCR product shown in Fig. 1c confirmed the centrin genecontaining sequence was precisely deleted at the predicted gRNA target sites and the chromosome fused through the donor sequence as intended (Fig. 1d). The gRNA/Cas9 expressing pLdCNa\&b plasmid was subsequently removed from the L. major centrin null mutant $\left(\mathrm{LmCen}^{-/-}\right)$by single-cell cloning and maintaining replica cultures in the presence and absence of G418 to identify clones sensitive to G418 that had lost the neomycin resistance gene present in the pLdCN CRISPR gene-editing plasmid. It was not possible to amplify plasmid DNA from the G418 sensitive $\mathrm{LmCen}^{-1-}$ parasite providing further evidence for the loss of the pLdCNa\&b plasmid (Supplementary Fig. 1B). As also shown in Supplementary Fig. 1C, the $\mathrm{LmCen}^{-1-}$ parasite retained the phenotype of slower proliferation than the WT $L$. major. This difference in proliferation enabled the identification and isolation of the slower growing centrin gene deleted clones by visual and microscopy inspection of the 96 well plate after one week in culture. 


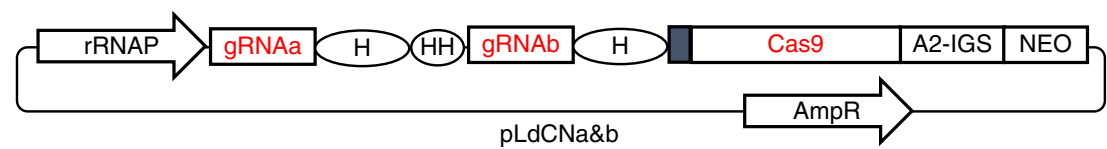

b

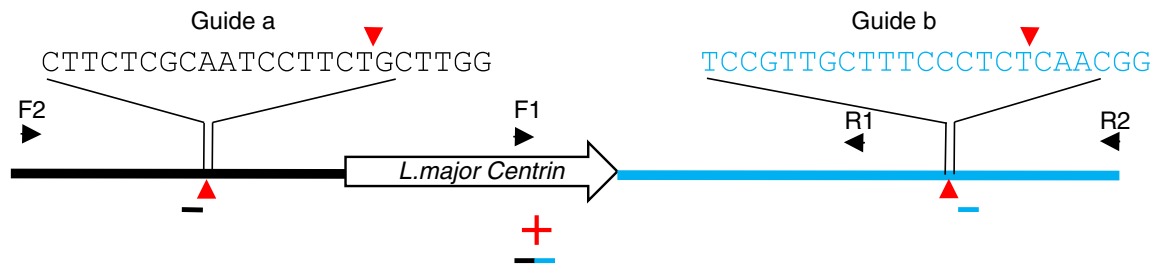

5’ ATtTCGTGCTTCTCGCAATCCTTCTCAACGGATGATAGTGCGCGTGTGCG

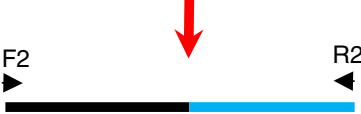

c

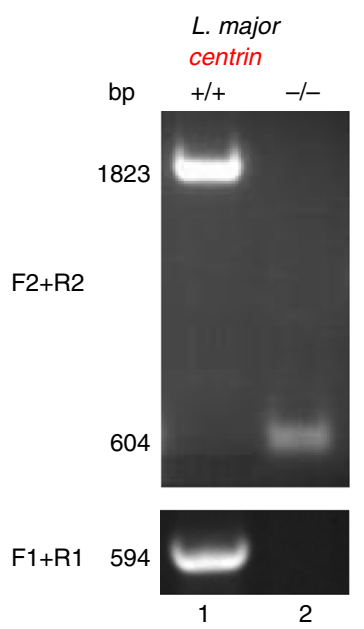

d

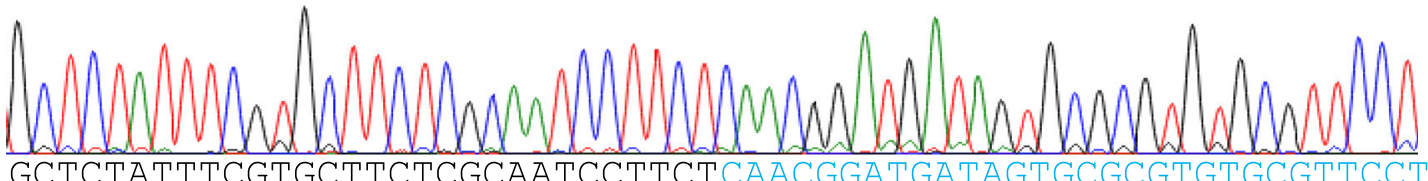
GCTCTATTTCGTGCTTCTCGCAATCCTTCTCAACGGATGATAGTGCGCGTGTGCGTTCCT

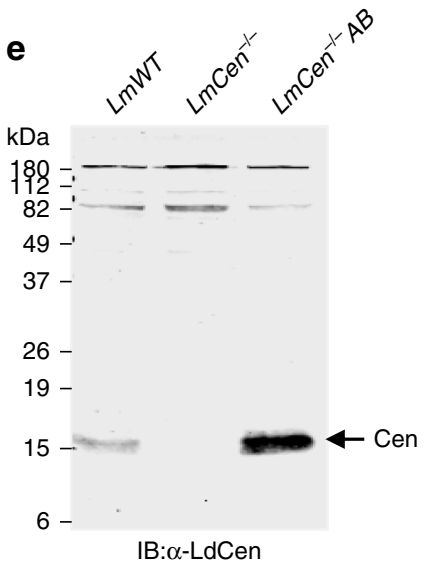

f

g
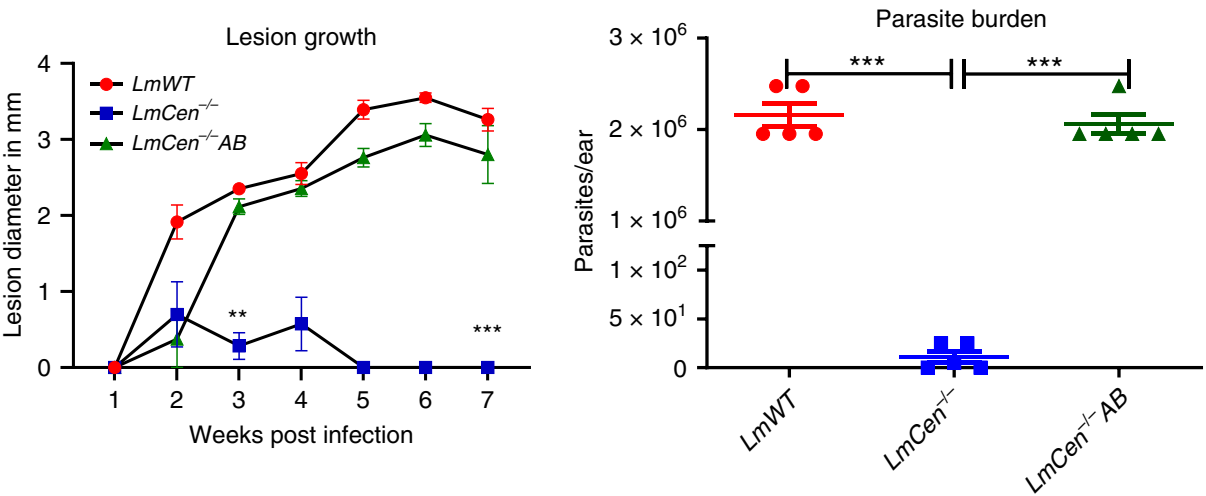

Fig. 1 Generation of marker free LmCen $^{-{ }^{-}-}$parasite. a The pLdCN vector used to express Cas9 and gRNAa and gRNAb in Leishmania. A2-IGS, L. donovani A2 gene intergenic sequence; rRNAP, L. donovani ribosomal RNA promoter; $\mathrm{H}$, Hepatitis delta virus ribozyme; $\mathrm{HH}$, Hammerhead ribozyme. b Schematic of gene deletion strategy showing gRNAa and gRNAb targeting sites in the L. major centrin gene locus (LmjF.22.1410) and the expected gene deletion sequence after transfection of the cells with a 50 nucleotide oligonucleotide donor. The primers F1-R1 and F2-R2 used to detect this deletion are indicated. c PCR analysis with primers F1-R1 and F2-R2 revealing loss of the centrin gene. Lane 1, Wildtype L. major; lane 2, L. major centrin null mutant. d Sequence analysis confirming the flanking DNA breaks joined together by the transfected 50 nucleotide oligonucleotide donor. See the supplementary information for the detailed sequence. e An immunoblot, representative of three independent experiments, with an $\alpha$-LdCentrin antibody showing the re-expression of Centrin in $\mathrm{LmCen}^{-/-}$parasites transfected with a pKSNeo- $\mathrm{LmCEN}$ plasmid ( $\mathrm{LmCen}^{-/-}$-AB, Addback). $\mathbf{f} \mathrm{LmCen}^{-/-}$was unable to induce ear cutaneous lesions in C57BL/6 mice compared to wildtype L. major or the centrin add-back parasites of $L m C e n^{-/-}$showing restored virulence (green line). C57BL/6 mice ( $n=5$ per group) were infected intradermally $\left(1 \times 10^{6}\right)$ with $L m W T, L m C e n^{-/-}$or $L m C e n^{-/-} A B$ parasites and the ear lesion development was monitored weekly. Data is plotted as mean \pm SEM. and is representative of two independent experiments. Unpaired two tailed Student's $t$ test was used to calculate statistical significance between $L m W T$ and $L m C e n^{-/-}$or $L m C e n^{-/}-A B$ and $L m C e n^{-/-}$groups $\left({ }^{\star \star} p<0.01,{ }^{\star \star \star} p<0.001\right)$. $\mathbf{g}$ Parasite load in the infected ears of the mice ( $n=5$ per group). Parasite burden was determined by limiting dilution assay. Data is plotted as mean \pm SEM. and is representative of two independent experiments. Unpaired two tailed Student's $t$ test was used to calculate statistical significance. Statistical analysis was performed by unpaired two-tailed t-test $\left({ }^{\star \star \star} p<0.001\right)$.

$\mathrm{LmCen}^{-1-}$ does not produce lesions in infected mice. It was necessary to establish whether the $\mathrm{LmCen}^{-1-}$ had lost the ability to cause cutaneous infections and whether adding back the centrin gene through plasmid transfection (add-back, $\mathrm{LmCen}^{-1-} \mathrm{AB}$ ) could restore cutaneous infection. The centrin gene was inserted into the Leishmania pKSNeo expression plasmid $^{36,37}$, transfected into $\mathrm{LmCen}^{-1-}$ promastigotes and expression of the centrin protein was confirmed by Western blotting with an a-LdCen antibody that can recognize L. major centrin (Fig. 1e). $\mathrm{LmCen}^{-1-}$ infection was investigated following intradermal injection of 
$1 \times 10^{6}$ stationary phase promastigotes in the ear of $\mathrm{C} 57 \mathrm{BL} / 6$ mice. As shown in Fig. 1f, by 5-6 weeks, $\mathrm{LmCen}^{-1-}$ failed to produce swelling in the infected ear whereas wildtype $L$. major ( $L m W T$ ) Friedlin V9 and the $L m \mathrm{Cen}^{-1-}$ with the add-back centrin gene $\left(\mathrm{LmCen}^{-1-} \mathrm{AB}\right)$ did induce significant swelling. At 6 weeks following infection, the $\mathrm{LmCen}{ }^{-1-}$ infected mice had few $(<10)$ detectable parasites compared to both the $\operatorname{LmWT}$ and $\mathrm{LmCen}^{-1-} A B$ infected mice that both had significantly more parasites $\left(\sim 2 \times 10^{6}\right)$ (Fig. 1g). These observations confirm that at 6 weeks post-infection, marker-free $\mathrm{LmCen}^{-1-}$ is unable to induce pathology at the site of injection in mice and that this was due to the deletion of the centrin gene.

We next examined $\mathrm{LmCen}{ }^{-1-}$ survival in human macrophages in vitro since these are the obligate host cells for intracellular replication of Leishmania amastigotes (Supplementary Fig. 1D). At $24 \mathrm{~h}$ post-infection, the number of parasites per macrophage was similar in the $\mathrm{LmCen}^{-1-}$ and $\mathrm{LmWT}$ infected cells. However, by 8 days, $\mathrm{LmCen}^{-1-}$ amastigotes were cleared from the macrophages, whereas $L m W T$ parasites reached >10 parasites/ macrophage. These results demonstrated that the $\mathrm{LmCen}^{-1-}$ promastigotes effectively infected human macrophages but subsequently were unable to proliferate intracellularly.

$\mathrm{LmCen}^{-/-}$contains no off-target gene deletions. Since the CRISPR generated $\mathrm{LmCen}^{-1-}$ strain was attenuated, it was necessary to establish the integrity of the genome by wholegenome sequencing analysis to confirm the attenuation seen was solely due to the removal of the centrin gene. This analysis confirmed that the targeted $\sim 1 \mathrm{~kb}$ genome region containing the 450 bp centrin gene (ID:LmjF.22.1410) was deleted from chromosome 22 and the remaining centrin gene homologs on chromosomes 7 , 32, 34 and 36 remained intact in the genome (Fig. 2a). Southern blot analysis confirmed the targeted centrin gene in $\mathrm{LmCen}^{-1-}$ was deleted and not translocated to another region of the genome (Fig. 2b). Whole-genome sequencing was performed to establish whether there were any off-target gene deletions in the edited genome. As shown in Fig. 2c, the blue line is comprised of over 8,000 circles, each circle representing a single gene from chromosome 1 through 36 (left to right) whereas the red circles represent the members of the centrin gene family located on chromosomes $7,22,32,34$ and 36 . There was virtually $100 \%$ coverage for all 8307 genes in the genome indicating the absence of partial or complete gene deletions, except the targeted centrin (LmjF.22.1410) that had a $0 \%$ coverage since it was deleted through CRISPR gene editing. A handful of genes (open blue circles) with less than $100 \%$ coverage are tandem repeat genes for which the coverage calculation software misaligned some reads, these genes were manually inspected and were found to be intact. Compared to the L. major Friedlin reference genome, there were no indels and no new SNPs (21 genes contained SNPs that were all previously identified in resequencing of the $L$. major Friedlin or LV39 strains). Collectively, these analyses demonstrate that the $\mathrm{LmCen}^{-1-}$ genome is intact and has no off-target gene mutations.

The genomic DNA sequence reads were also searched for the presence of pLdCN CRISPR plasmid DNA sequence to confirm the loss of this plasmid. As shown in Fig. 2d, the only $\mathrm{LmCen}^{-1-}$ genomic DNA sequence in common with the pLdCN CRISPR plasmid was the A2 gene intergenic sequence (A2-IGS) that is part of a A2 pseudogene sequence present in the L. major genome. The A2-IGS sequence from $L$. donovani was incorporated into the pLdCN CRISPR plasmid for processing of the $\mathrm{Neo}^{\mathrm{R}}$ gene transcript ${ }^{32}$. There were no other detectable plasmid sequences or antibiotic resistance genes in the genome of $\mathrm{LmCen}^{-1-}$. It is noteworthy that the L. donovani ribosomal RNA promoter (rRNAP) sequence in the pLdCN CRISPR plasmid is sufficiently divergent from the L. major rRNAP sequence that it was not identified in the MiSeq DNA sequences by the Maximal Exact Match (bwa-mem) sequence alignment algorithm used. Taken together, the results presented in Fig. $2 \mathrm{~d}$ and Supplementary Fig. 1B in combination with the loss of G418 resistance demonstrate that the pLdCN CRISPR gene-editing plasmid is no longer present in $\mathrm{LmCen}^{-1-}$. This represents a significant milestone since $L m C e n^{-1-}$ is the first marker-free gene deleted Leishmania strain to be generated in the laboratory.

$\mathrm{LmCen}^{-/-}$is safe and does not cause lesions in mice. As shown in Fig. 1, $\mathrm{LmCen}^{-1-}$ was unable to induce ear cutaneous lesions in C57BL/6 mice due to the removal of the centrin gene. However, to assess the safety of $\mathrm{LmCen}^{-1-}$ as a potential live vaccine, it was necessary to investigate its attenuation in a more susceptible mouse strain $(\mathrm{BALB} / \mathrm{c})$ and in immune-deficient mice. $\mathrm{BALB} / \mathrm{c}$ mice injected subcutaneously in the footpad with $1 \times 10^{7}$ stationary phase $\mathrm{LmCen}^{-1-}$ showed no footpad swelling over 20 weeks (Fig. 3a), the study endpoint, and a significantly lower parasite burden (approximately 4 log fold reduction) as compared to $\mathrm{BALB} / \mathrm{c}$ mice injected with $L m W T$ (Fig. 3a). In some animals $\mathrm{LmCen}^{-1-}$ parasites were completely cleared by the study end point. Likewise, STAT-1 KO immune-deficient mice injected with $2 \times 10^{8} \mathrm{LmCen}^{-1-}$ stationary phase parasites showed no footpad swelling during 7 weeks following injection (Fig. 3b) whereas footpad swelling started at 4 weeks after injection with $L m W T$ (Fig. 3b). The parasite burden at 7 weeks in STAT-1 KO mice injected with $L m W T$ was significantly higher than the mice injected with $\mathrm{LmCen}^{-1-}$ attenuated parasites ( $\sim 6 \mathrm{log}$ fold reduction, Fig. 3b). In another test, IFN- $\gamma \mathrm{KO}$ mice showed severe footpad swelling accompanied by a drastic increase in the number of the parasites after injection with $1 \times 10^{7} \mathrm{LmWT}$, while injection with the same dose of $\mathrm{LmCen}^{-1-}$ did not show any footpad swelling in 20 weeks and the parasites were cleared from the site of injection (Fig. 3c). The recombination activating gene 2 deficient (Rag2 KO) mice, which lack conventional T cells and B cells, showed mild footpad swelling and a high parasite burden in the footpad after 15 weeks following injection with $L m W T$ (Fig. 3d). In contrast, injection with $\mathrm{LmCen}^{-/-}$did not show any swelling (Fig. 3d; Supplementary Fig. 2C) and the parasites were cleared from the site of injection (Fig. 3d) and the spleen and liver (Supplementary Fig. 2D). These results demonstrate that $\mathrm{LmCen}^{-\mathrm{T}}$ is non-pathogenic even in highly immunocompromised mice.

To rule out the survival of any undetectable $\mathrm{LmCen}^{-1-}$ parasites beyond 7 weeks post-immunization, $\mathrm{LmCen}^{-/-}$infected $\mathrm{BALB} / \mathrm{c}$ mice were treated with $2 \mathrm{mg} / \mathrm{kg}$ dexamethasone (DXM), a known immune suppressor, three times for a week starting at 10 weeks post-infection (Fig. 3e). All the groups were sacrificed at 4 weeks after the DXM treatment to determine parasite burdens. As shown in Fig. $3 \mathrm{f}$ and Supplementary Fig. 2A, $\mathrm{LmCen}^{-1-}$ infected mice with or without DXM treatment resulted in no lesions while $L m W T$ infected but DXM-untreated mice developed open ulcerative lesions in the ear. Moreover, only 2 of 12 DXMtreated mice infected with $\mathrm{LmCen}^{-1-}$ showed parasites in the inoculated ear (Fig. 3g) and draining lymph node (Fig. 3h). In 1 of 6 untreated $\mathrm{LmCen}^{-1-}$-immunized animals, a low parasite number was detected in the draining lymph node $(<100$ parasites, Fig. $3 \mathrm{~h}$ ), and none in the ear (Fig. 3g). In $L m W T$ infected mice, a significantly higher parasite load was observed in the ear and draining lymph node compared to $\mathrm{LmCen}^{-1-}$-infected mice $( \pm$ DXM) (Fig. $3 \mathrm{~g}, \mathrm{~h}$ ), which correlated with ear lesion size (Fig. 3f). Further, a PCR analysis using L. major centrin genespecific primers confirmed the absence of the centrin gene in the parasites isolated from DXM-treated mice (Supplementary Fig. 2B 
a

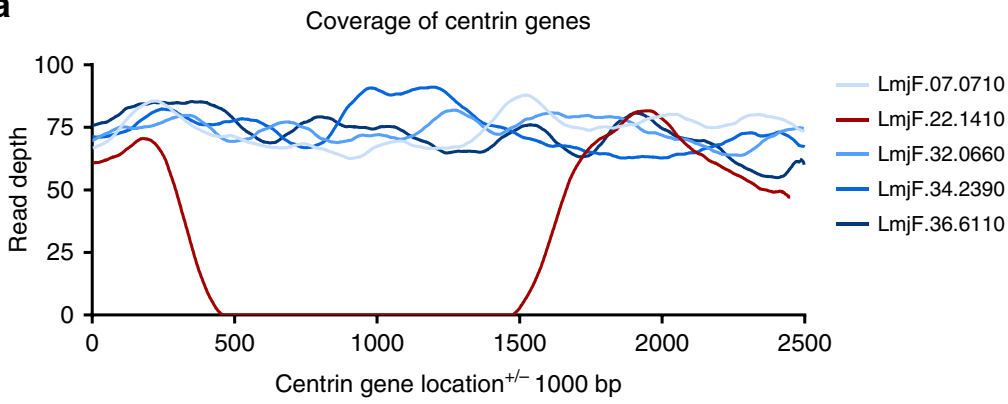

C

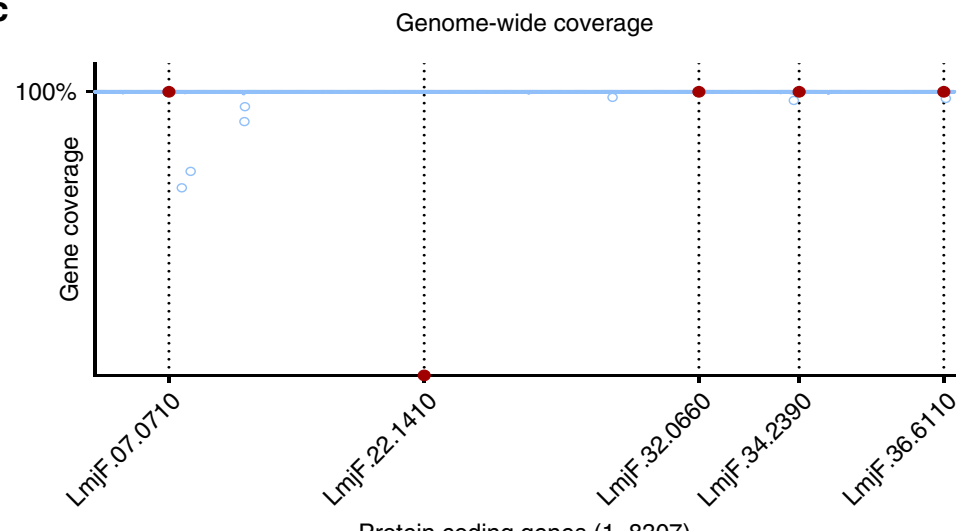

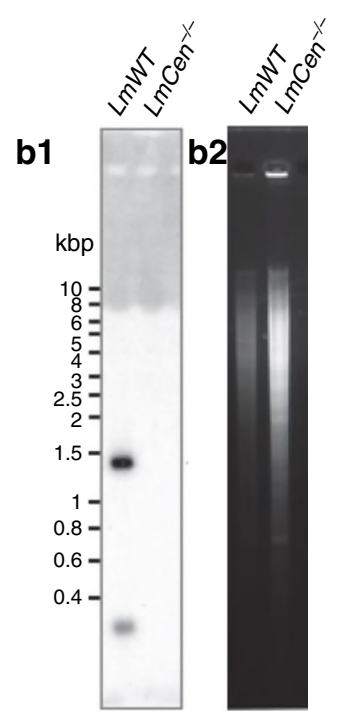

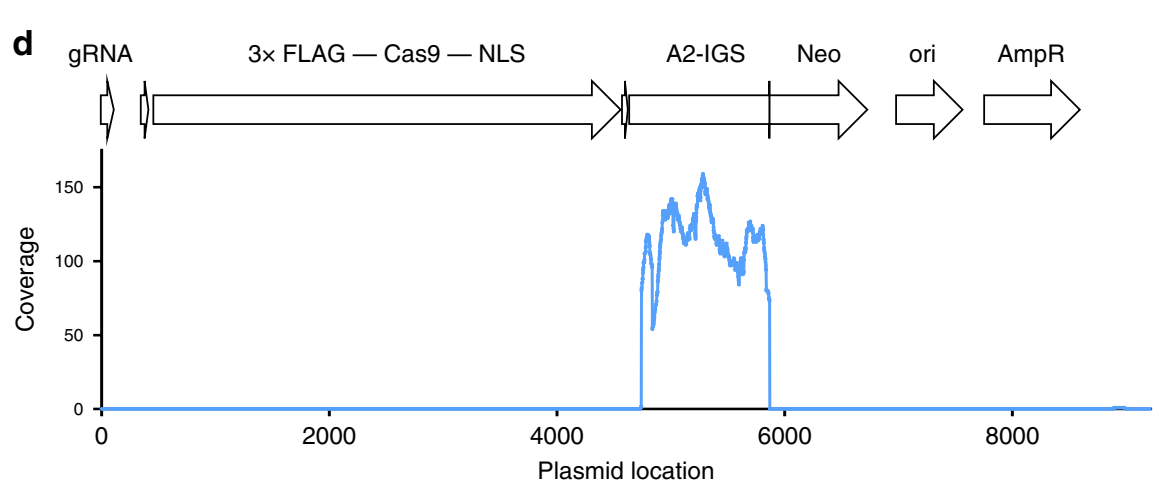

Fig. 2 Whole-genome analysis of the attenuated $\mathbf{L m C e n}{ }^{-1}-$ L. major. a Sequence coverage across each of the centrin gene family members in the $\mathrm{LmCen}^{-/-}$L. major. Note that only the targeted LmjF.22.1410 centrin gene has no sequence reads resulting from CRISPR gene editing. b1-b2 Southern blot analysis (b1) revealing the absence of the LmjF.22.1410 centrin gene in the genome of $L m C e n^{-/-}$parasite compared to wildtype $L$. major, $L m W T$. The ethidium bromide-stained agarose gel (b2) showing the Bglll digested genomic DNA from LmWT and $L m C e n^{-/-}$. c Percent sequence coverage ( $Y$-axis) for all protein-coding genes from chromosome 1 to 36 (X-axis) by Illumina sequencing of the whole genome of the $L m C e n^{-/-} L$. major. The blue line across the $X$ axis is composed of 8307 dots where each dot represents a gene starting from chromosome 1 (left) to chromosome 36 (right) and is placed according to the portion of the open reading frame supported by sequencing reads. Open blue circles indicate genes where misalignments of sequencing Illumina reads occurred for some multicopy genes, although these genes were verified to be intact. Red circles and line markers correspond to the 5 centrin genes across the genome in chromosomes 7, 22, 32, 34 and 36. Only the targeted centrin gene (LmjF.22.1410) has been deleted from the genome and therefore has 0\% coverage. d Coverage of the pLdCN CRISPR plasmid sequence generated from whole-genome sequencing. No homologous plasmid sequences were detected in the $\mathrm{LmCen}^{-/-}$genome except for the positions $\sim 5000$ to 6000 corresponding to the Leishmania donovani A2 gene intergenic sequence (A2-IGS) that were incorporated into the pLdCN plasmid for expression of the Neo ${ }^{R}$ gene. Therefore, the A2-IGS genomic sequence reads can align to this portion of the plasmid although the pLdCN CRISPR plasmid is not present in $\mathrm{LmCen}^{-/-}$.

lane 1, red arrow). Collectively, these results revealed that the centrin deleted live $\mathrm{LmCen}^{-1-}$ parasites are unable to revert or cause pathology and are safe for further study as a live vaccine.

$L m C e n^{-/-}$immunization protects against wildtype L. major. To investigate the protective efficacy of $\mathrm{LmCen}^{-1-}$ against wildtype L. major, both resistant (C57BL/6) and susceptible (BALB/c) mice were immunized with a single intradermal (i.d.) injection with $1 \times 10^{6}$ stationary phase $\mathrm{LmCen}^{-1-}$ in one ear. Seven weeks post-immunization, mice were challenged with 750 metacyclic wildtype L. major (WR 2885 strain) parasites in the contralateral ear via the i.d. route (Fig. 4a for C57BL/6; Supplementary Fig. 3A for BALB/c). Following challenge with wildtype L. major, lesion development was assessed up to 10 weeks for the C57BL/6 mice (Fig. 4b, c). In the non-immunized-challenged group, mice developed a non-healing open ulcer that progressively increased 
a

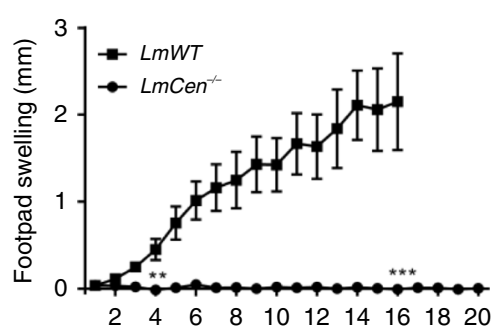

C

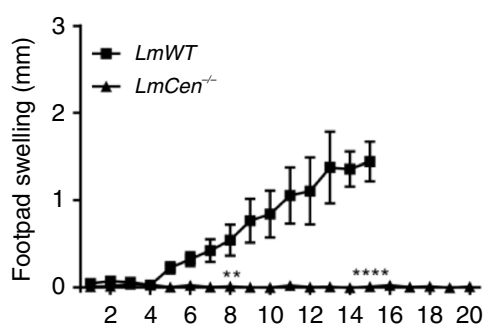

e

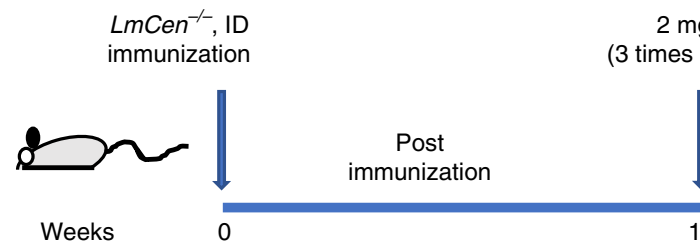

f

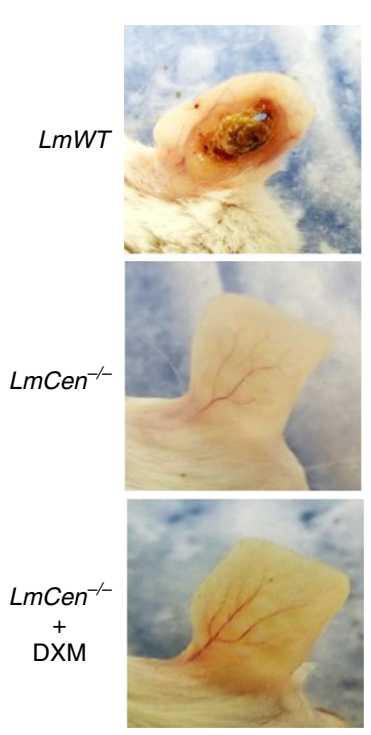

g

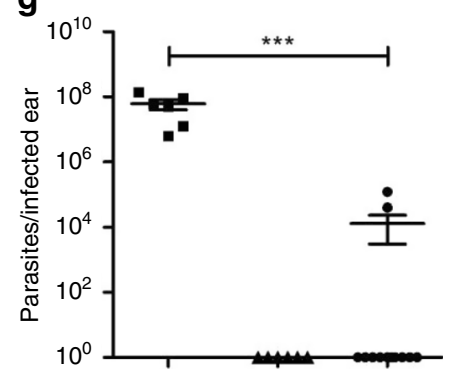

b
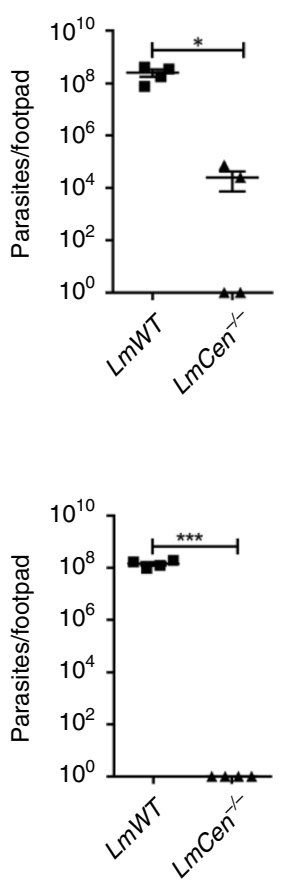
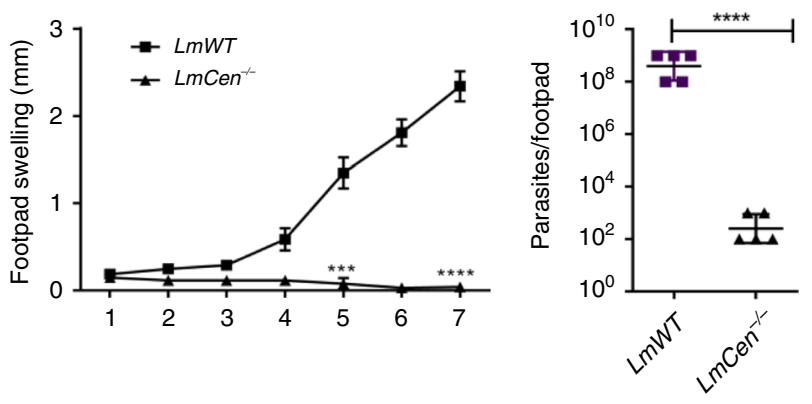

d

STAT1 KO

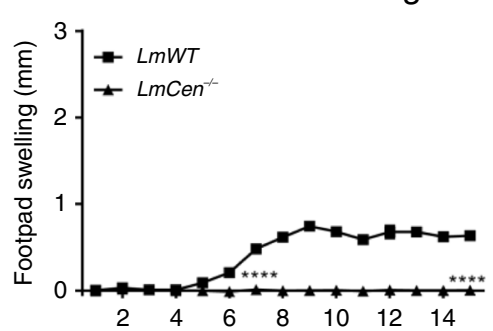

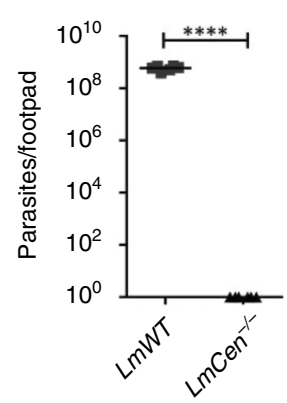

$\mathrm{mg} / \mathrm{kg}$ DXM treatment (3 times S.C injection for a week)

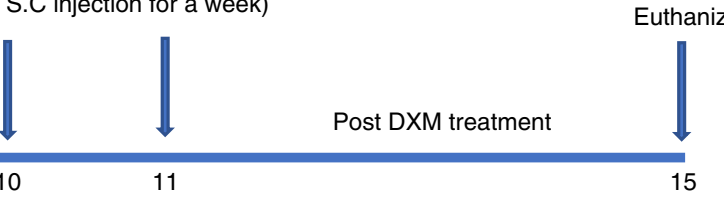

h

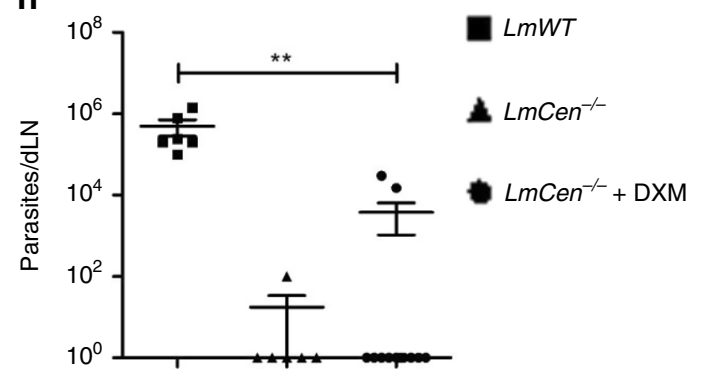

in size (Fig. 4c). No open ulcers were observed in the $\mathrm{LmCen}^{-1-}$-immunized-challenged group and only a moderate swelling that subsided from 5 to 9 weeks post-challenge was observed in 6 of 13 mice. Figure $4 \mathrm{c}$ depicts the ear pathology at 10 weeks post-challenge compared to a naïve unchallenged ear. Importantly, histopathological analysis revealed no clear difference between immunized-challenged and naïve mice ears, while non-immunized-challenged mice ears developed large lesions with open ulcers involving an influx of inflammatory cells
(Fig. 4c). The parasite load in the challenged ear and draining lymph node were also quantified at 10 weeks post-challenge revealing that the immunized group had a significantly lower parasite load (approximately a 4-log fold and a 3.2-log fold reduction, respectively) compared to the non-immunized group (Fig. 4d, e). Similarly, highly susceptible BALB/c mice were protected following immunization with $\mathrm{LmCen}^{-1-}$ parasites (Supplementary Fig. 3A-E). At 10 weeks post-challenge with wildtype $L$. major parasites, immunized BALB/c mice were 
Fig. 3 Safety and non-pathogenicity characteristics of $\mathbf{L m C e n}^{-/-}$parasites. a BALB/c; b STAT1 KO; $\mathbf{c}$ IFN- $\gamma$ KO and $\mathbf{d}$ Rag2 $\mathrm{KO}$ mice were subcutaneously inoculated with indicated doses of $L m W T$ or $L m C e n^{-/-}$into the right hind footpad. a BALB/c mice $(L m W T, n=5)$ or $\left(L m C e n^{-/-}, n=7\right)$ c IFN- $\gamma \mathrm{KO}$ mice $(L m W T, n=4)$ or $\left(L m C e n^{-/-}, n=6\right)$, d Rag2 KO mice $(L m W T, n=6)$ or $\left(L m C e n^{-/-}, n=6\right)$ were infected with $1 \times 10^{7}$ of $L m W T($ Friedlin V9) or $\mathrm{LmCen}^{-/-}$and $\mathbf{b}$ STAT1 KO mice were infected with $2 \times 10^{8}$ of $\operatorname{LmWT}$ (Friedlin V9) $(n=4)$ or $\mathrm{LmCen}^{-/}-(n=4)$ parasites. Following infection, footpad swelling was measured weekly by digital caliper. Parasite burden in infected footpad was measured at 5 weeks after infection in BALB/c (LmWT,

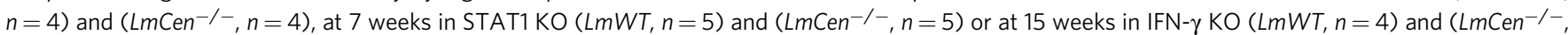
$n=4)$ and at 15 weeks in Rag2 KO (LmWT, $n=6)$ and $\left(L m C e n^{-/-}, n=6\right)$. For the lesion development studies shown in a-d (left panels) as SEM a, c and $\mathbf{d}$ and as SD $\mathbf{b}$ and for parasite burden studies shown in $\mathbf{a}$-d (right panel) as mean \pm SEM a, $\mathbf{c}$ and $\mathbf{d}$ and as mean \pm SD $\mathbf{b}$, unpaired two-tailed t-test $\left({ }^{\star} p-0.04,{ }^{\star \star} p-0.009,{ }^{\star \star *} p<0.0009,{ }^{\star \star \star *} p<0.0001\right)$. The differences in footpad swelling were statistically significant at all time points after the initial observation of the lesion. Experiments representing $\mathbf{a}, \mathbf{b}$ and $\mathbf{c}$ performed twice; and experiment representing $\mathbf{d}$ performed only one time. $\mathbf{e}$ Schematic representation of the DXM treatment. $\mathbf{f}$ Photographs of one representative ear of $L m W T$ infected (group- 1$)(n=6), L m C e n^{-/-}(g r o u p-2)(n=6)$ and $\left(\mathrm{LmCen}^{-/-}+\mathrm{DXM}\right)$ (group-3) $(n=12)$ mice from one of two independent experiments. Scatter dot plots of parasite load in the infected ear $\mathbf{g}$ and in the draining lymph node $(\mathrm{dLN}) \mathbf{h}$ of each $\operatorname{LmWT}(n=6)$ and $\mathrm{LmCen}^{-/-}(n=6)$ and $\operatorname{LmCen}{ }^{-/-}+\mathrm{DXM}(n=12)$ immunized mice. Parasite burden was determined by limiting dilution assay. Results are expressed as mean \pm SEM and data pooled from two independent experiments. Statistical analysis was performed by unpaired two-tailed t-test $\left({ }^{\star \star} p-0.004,{ }^{\star \star \star} p-0.0006\right)$.

a

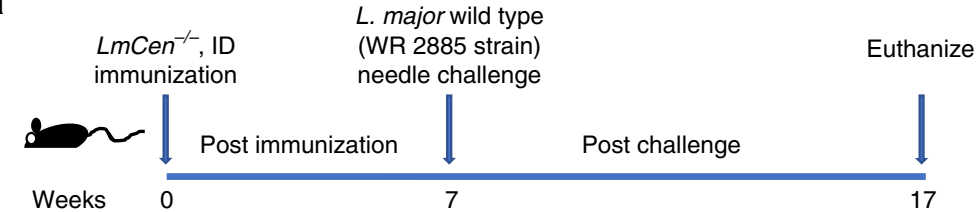

b

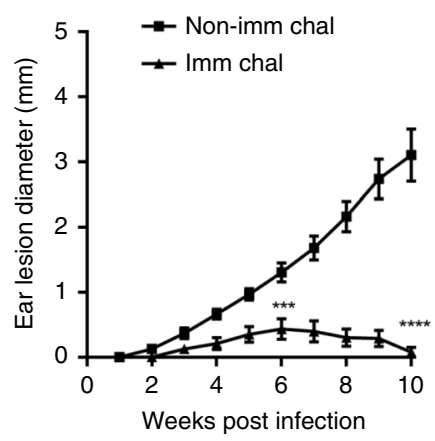

d

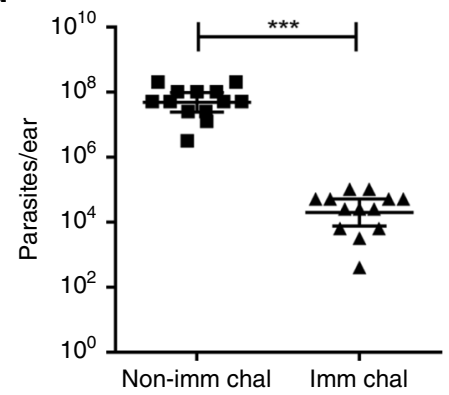

C

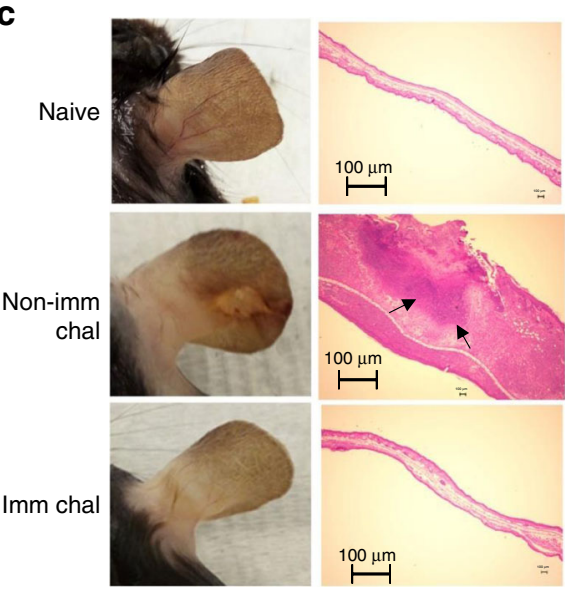

e

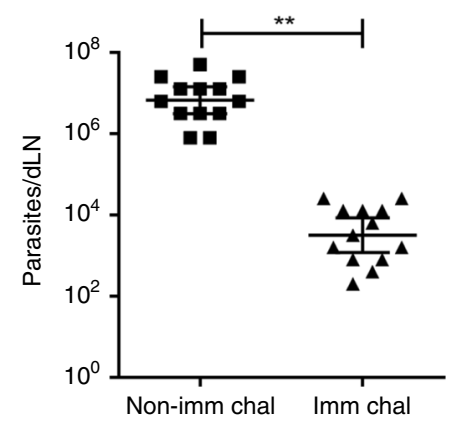

Fig. 4 Protective efficacy of $\mathbf{L} \mathbf{m C e n}{ }^{-/-}$parasites against virulent $\boldsymbol{L}$. major needle challenge in C57BL/6 mice. a Schematic representation of the needle challenge procedure. $\mathbf{b}$ Ear lesion diameter was measured weekly for both $L m C e n^{-1-}$ immunized (Imm Chal) $(n=13)$ and non-immunized (Non-Imm Chal) $(n=13)$ mice after intradermal challenge with $L m W T$ parasites. Results are represented as SEM, of 1 ear from each of the 13 mice per group and data pooled from two independent experiments. Statistical analysis was performed by unpaired two-tailed t-test $\left({ }^{\star \star \star} p-0.0005,{ }^{\star \star \star *} p<0.0001\right)$. c Photographs (left panel) \& histology (H\&E stained, right panel) of representative ear of age-matched naïve, LmCen ${ }^{-/-}$immunized (Imm Chal) \& non- immunized (NonImm Chal) mice after 10 weeks post challenge from one of two independent experiments $(n=2)$. Ear lesion histology sections are representative of one mouse from each group of one of two independent experiments. Arrow indicates inflammatory cells recruited area. Scale Bars: $100 \mu \mathrm{m}$. $\mathbf{d}$ and $\mathbf{e}, \mathrm{Scatter}$ dot plots of parasite load in the challenged ears (d) and in the draining lymph nodes (e) of each $\mathrm{LmCen}^{-/-}$immunized (Imm Chal) ( $\left.\mathrm{n}=13\right) \&$ non-Immunized (Non-Imm chal) $(n=13)$ mice. Parasite burden was determined by limiting dilution assay. Results are mean \pm SEM. Data are pooled from two independent experiments. Statistical analysis was performed by unpaired two-tailed t-test $\left({ }^{\star \star} p-0.004,{ }^{\star \star \star} p-0.0004\right)$. 
a

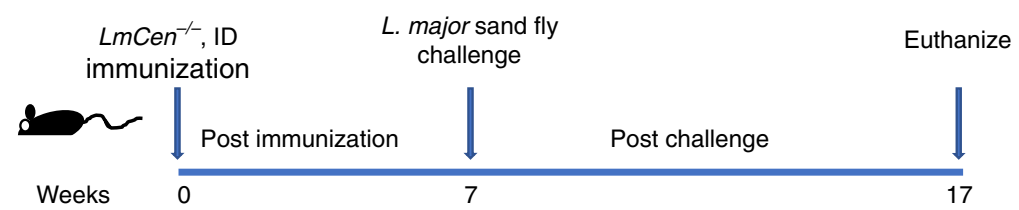

b

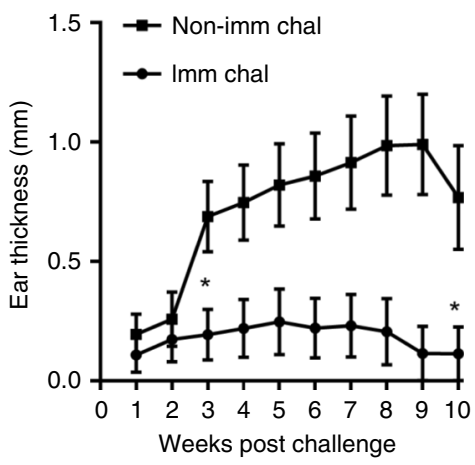

d

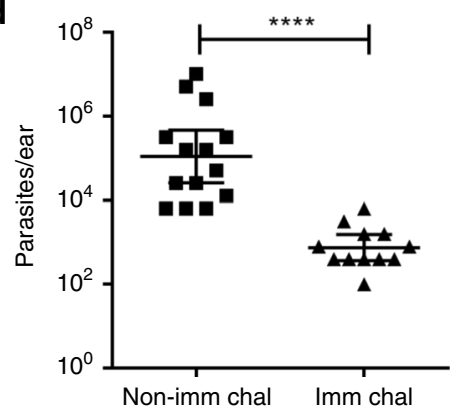

C

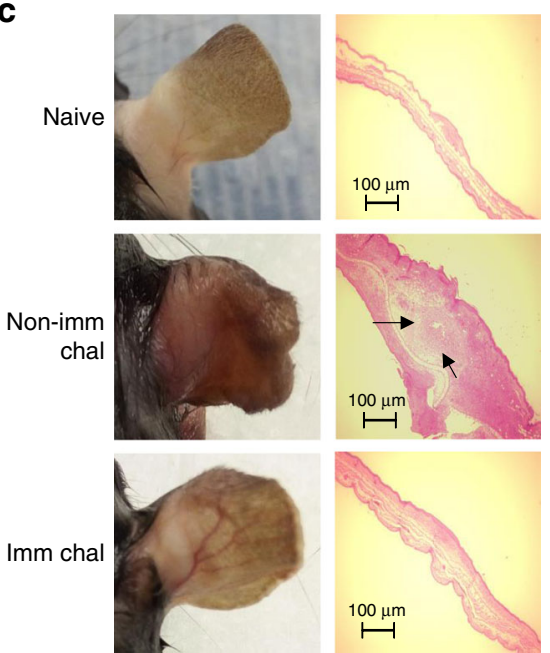

e

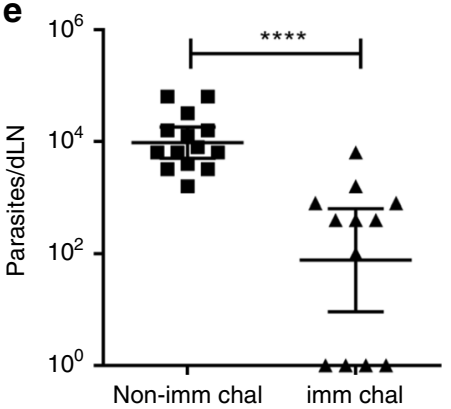

Fig. 5 Protective efficacy of $\mathbf{L m C e n}{ }^{-/}$parasites against sand fly challenge in C57BL/6 mice. a Schematic representation of the sand fly challenge procedure. $\mathbf{b}$ Ear lesion thickness was measured weekly for both $\mathrm{LmCen}-/-$ immunized (Imm Chal) $(n=12)$ and non-immunized (Non-Imm Chal) ( $n=14)$ mice after sand fly transmission. only 1 mouse out of $12 \mathrm{LmCen}^{-/-}$immunized-challenged has developed severe lesion. Results are represented as SEM, of 1 ear from each of the 12-14 mice per group and data pooled from two independent experiments. Statistical analysis was performed by Mann-Whitney twotailed test $\left({ }^{\star} p-0.02\right)$. c Photographs (left panel) \& histology (H\&E staining right panel) of representative ear of age-matched naïve, $L m C e{ }^{-} /-$immunized (Imm Chal) \& non- immunized (Non-Imm Chal) mice after 10 weeks post challenge from one of two independent experiments. Ear histology sections are representative of one mouse from each group of one of two independent experiments $(n=2)$. Arrow indicates inflammatory cells recruited area. Scale Bars: $100 \mu \mathrm{m}$. Scatter dot plots of parasite load in the challenged ear (d) and in the draining lymph node (e) of each $\mathrm{LmCen}{ }^{-/-}$immunized (Imm Chal) $(n=$ 12) \& non-Immunized (Non-Imm chal) $(n=14)$ mice. Parasite burden was determined by limiting dilution assay. Results are represented as geometric means with $95 \% \mathrm{Cl}$ of total $12-14$ mice in each group. Data are pooled from two independent experiments. Statistical analysis was performed by Mann-Whitney two-tailed test $\left({ }^{\star \star \star *} p<0.0001\right)$.

protected as measured both by a reduced lesion size (Supplementary Fig. 3B, C) and parasite burden (Supplementary Fig. 3D, E) compared to non-immunized-challenged mice. A similar lack of non-healing open ulcer was observed in $\mathrm{LmCen}^{-1-}$ immunized $\mathrm{BALB} / \mathrm{c}$ mice challenged with other wildtype strains of $L$. major such as L. major FV9 (Supplementary Fig. 3F) and L. major LV39 (Supplementary Fig. 3G).

$\mathrm{LmCen}^{-/-}$protects against sand fly transmitted L. major WT. It is substantially more difficult and more relevant to demonstrate immunological protection against L. major infection initiated by a sandfly challenge than by a needle injection challenge $e^{20,38}$. Therefore, to determine the efficacy of $\mathrm{LmCen}^{-1-}$ immunization against sand fly transmitted cutaneous infection by L. major,
C57BL/6 mice were immunized with a single i.d. injection of $1 \times$ $10^{6} \mathrm{LmCen}^{-1-}$ stationary phase parasites and mice were infected by exposure to bites of $10 \mathrm{~L}$. major-infected sand flies in the contralateral ear 7 weeks post-immunization (Fig. 5a). Disease progression was monitored for 10 weeks post-challenge by measuring lesion growth and assessing parasite burden in the ear and draining lymph node (Fig. 5b-e). Notably, only 1/12 immunized-challenged mice developed a visible lesion, while 10/ 14 non-immunized-challenged mice developed progressive lesions in the ear that were significantly larger than the single lesion observed in immunized-challenged mice (Fig. 5b, c). At 10 weeks post-challenge, there was a significant reduction of the parasite burden both in the ear and draining lymph node (approximately $2 \mathrm{log}$ fold reduction in both) of immunizedchallenged mice compared to non-immunized-challenged mice 
(Fig. 5d, e). It is interesting to note that some of the draining lymph nodes in the immunized-challenged mice did not have any parasites (Fig. 5e). These results demonstrate that immunization with $\mathrm{LmCen}^{-1-}$ mediates significant protection under natural conditions of infection i.e., parasite transmission by an infected sand fly.

$\mathrm{LmCen}^{-/-}$or leishmanization induced similar immunological protection. Previously, in murine leishmanization models, it was shown that leishmanization induces host protective immunity against re-infection ${ }^{19,20}$. Having shown above that $\mathrm{LmCen}^{-1-}$ induces protection against both needle and the natural model of sand fly challenge, we first compared the immune response between $\mathrm{LmCen}^{-1-}$ immunized group (8 weeks of post-immunization) and a primary $L m W T$ infection (healed group) at 12weeks of post-primary infection (Fig. 6a). The rationale for using two different time points is in leishmanization group, mice infected with wild type L. major causes lesion development from 4 to 8 weeks and by $10-12$ weeks most of the lesions are healed and very few parasites are present. On the contrary in the case of $\mathrm{LmCen}^{-1-}$ parasite immunized animals they do not develop any active lesions, and beyond 6 weeks post-immunization we do not recover $\mathrm{LmCen}^{-1-}$ parasites in most mice.

The ears from the healed group showed lesions that later resolved. In the $\mathrm{LmCen}^{-1-}$ immunized group however, no lesion development was observed (Supplementary Fig. 4A, B). Antigen experienced $\mathrm{CD} 4{ }^{+} \mathrm{T}$ cells were first gated based on their surface expression of CD44 and $\mathrm{CD} 4{ }^{+} \mathrm{CD} 44^{+}$cells were rearranged into different subpopulations based on their production of TNF- $\alpha$, IFN- $\gamma$, and IL-2 (Fig. 6b). Fluorescence minus one (FMO) control was used for proper gating of positive events for designated cytokines (Supplementary Fig. 4C). The results showed that both $\mathrm{LmCen}^{-1-}$ immunization and healed groups of mice induced comparable single as well as multiple cytokines secreting $\mathrm{CD} 4{ }^{+} \mathrm{CD} 44^{+} \mathrm{T}$ cells upon re-stimulation with $L$. major freezethaw antigen ( $L m F T A g$ ) (Fig. 6c, d). Naïve mice (not immunized with $L m C e n^{-1-}$ or infected with $L m W T$ ) did not show any detectable immune response after antigen stimulation (Fig. 6c). Upon challenge with wildtype $L$. major parasites by needle injection, at $20 \mathrm{~h}$ post-infection, we observed a significant increase in the mRNA levels of IFN- $\gamma$ in both healed and $\mathrm{LmCen}^{-1-}$ immunized ear tissues compared to non-immunized mice (Fig. 6g). From the same time point after challenge $(20 \mathrm{~h}$ postinfection), we also analyzed the IFN- $\gamma$ production from effector CD4 $\mathrm{T}$ cells by flow cytometry. Both healed and $\mathrm{LmCen}^{-1-}$ immunized mice induced a significantly higher percentage of IFN- $\gamma^{+}$effector $\mathrm{T}$ cells (Live $\mathrm{CD} 4^{+} \mathrm{CD} 44^{\mathrm{Hi}} \mathrm{Ly}_{6 \mathrm{C}}{ }^{+} \mathrm{T}$-bet ${ }^{+}$) compared to the non-immunized group (Fig. 6f). Figure 6e shows common gating strategies for early immune response (Live $\mathrm{CD} 4{ }^{+} \mathrm{CD} 44^{\mathrm{Hi}}-\mathrm{Ly}_{6 \mathrm{C}}{ }^{+}$T-bet ${ }^{+} \mathrm{IFN}-\gamma^{+}-\mathrm{T}$ cells) in the ear of nonimmunized, healed and $\mathrm{LmCen}^{-1-}$ immunized mice at $20 \mathrm{~h}$ post needle challenge with wildtype $L$. major parasites. FMO control was used for proper gating of positive events for designated cytokines (Supplementary Fig. 4D).

Healed and $\mathrm{LmCen}^{-1-}$ immunized groups were also challenged with $L$. major WT infected sand fly and the parasite loads were determined (Fig. 6h, i). After five weeks of post-challenge, there was a similar significant reduction of parasite burden in the ear (2.4 log fold in healed group, and $2.1 \mathrm{log}$ fold in $\mathrm{LmCen}^{-1-}$ immunized group) and draining lymph nodes (1.7 log fold in healed group, and $1.48 \mathrm{log}$ fold in $\mathrm{LmCen}^{-1-}$ immunized group) compared to non-immunized group (Fig. 6h, i). Both healed and $\mathrm{LmCen}^{-1-}$ immunized-challenged mice did not develop any lesions whereas non-immunized-challenged mice developed cutaneous lesions (Supplementary Fig. 4E). Taken together, these results demonstrate that $\mathrm{LmCen}^{-1-}$ immunization is as effective as leishmanization ( $L m W T$ infection/healed) in generating a protective immune response and protecting against sand fly mediated infection with WT L. major.

\section{Discussion}

Leishmanization with wildtype $L$. major has so far been the only successful human vaccine for leishmaniasis but it is discontinued due to safety concerns associated with administration of a live virulent organisms. This paper describes a second generation leishmanization live vaccination with an attenuated L. major strain $\left(\mathrm{LmCen}^{-1-}\right)$ that does not cause lesions but retains the ability to provide immunological protection against experimental needle and sand fly transmitted Leishmania infection. As $\mathrm{LmCen}{ }^{-1-}$ is marker gene free safe and efficacious, can be advanced to Phase I human clinical trials.

CRISPR-Cas genome editing was essential to generating this marker-free strain because this technology can delete genes with high specificity and fidelity without selection with antibioticresistant marker genes ${ }^{32-34}$. In place of antibiotic marker selection, the selection was based on a reduced proliferation rate of the $\mathrm{LmCen}^{-1-}$ mutant identified through single-cell cloning, the first time such a selection has been performed in Leishmania. Wholegenome sequence analysis confirmed that only the centrin gene on chromosome 22 (ID:LmjF.22.1410) was precisely deleted at the CRISPR guide RNA targeting sites and other centrin gene members on chromosomes 7, 32, 34 and 36 remained intact. There were no CRISPR-induced off-target gene deletions, indels or nonsynonymous SNPs introduced in the $\mathrm{LmCen}^{-1-}$ clone that was subjected to whole-genome sequencing. By comparison, a previously engineered $L$. donovani centrin gene deleted parasites generated by homologous recombination with antibiotic-resistant marker genes did contain off-target genomic deletions of up to 5000 base pairs in non-coding regions and in the coding regions of the folate transporter and gp63 genes ${ }^{39}$. Although genetargeting specificity will depend largely on the selection of the guide RNA sequence, these observations suggest that CRISPRCas gene editing in Leishmania using a donor DNA fragment for repair as detailed in Fig. 1 is more specific than traditional homologous recombination-based gene replacement with antibiotic resistance markers. In theory, it could have also been possible to generate a centrin gene deleted markerless L. major parasite using a different CRISPR approach involving the transfection of recombinant SaCas9 protein with in vitro -transcribed guide RNAs directed to upstream and downstream sequences flanking the centrin gene ${ }^{40}$. Although deletion of other Leishmania virulence genes may likewise generate attenuated strains, the centrin gene was targeted in this study because centrin gene deleted $L$. donovani parasites have been the most extensively validated parasites in previous experimental vaccine studies using various animal models ${ }^{26-31}$. It is noteworthy that in this study, $L$. major was used instead of the previous studies involving centrin deleted $L$. donovani ${ }^{26-31}$ as the focus of this study was cutaneous leishmaniasis.

Numerous experimental vaccines have been developed for Leishmania, though most of them have not been tested against natural sand fly transmitted infections. In studies when such vaccines were tested by needle challenge versus sand fly transmission of a virulent parasite, they were either partially protective or not protective against the latter ${ }^{20,38,41,42}$. In addition, sand fly mediated infection provides other components present in the saliva which play an important role in the pathogenesis of Leishmania ${ }^{43-45}$. The observations reported here demonstrated that markerless $\mathrm{LmCen}^{-1-}$ immunization did induce protection against sand fly transmitted L. major. In this study, a major 
a

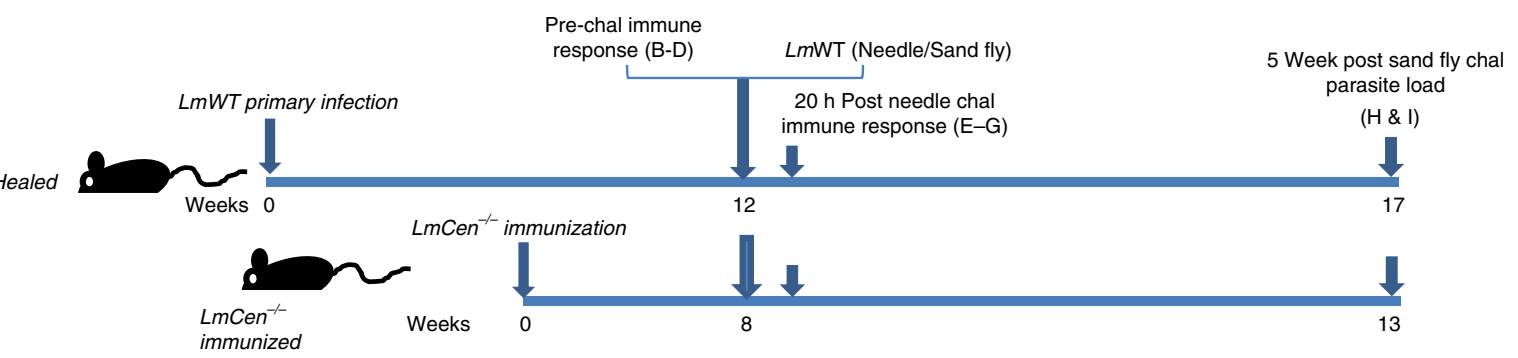

b

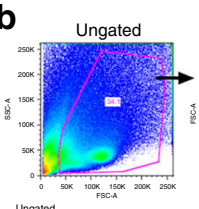

immunized

Weeks
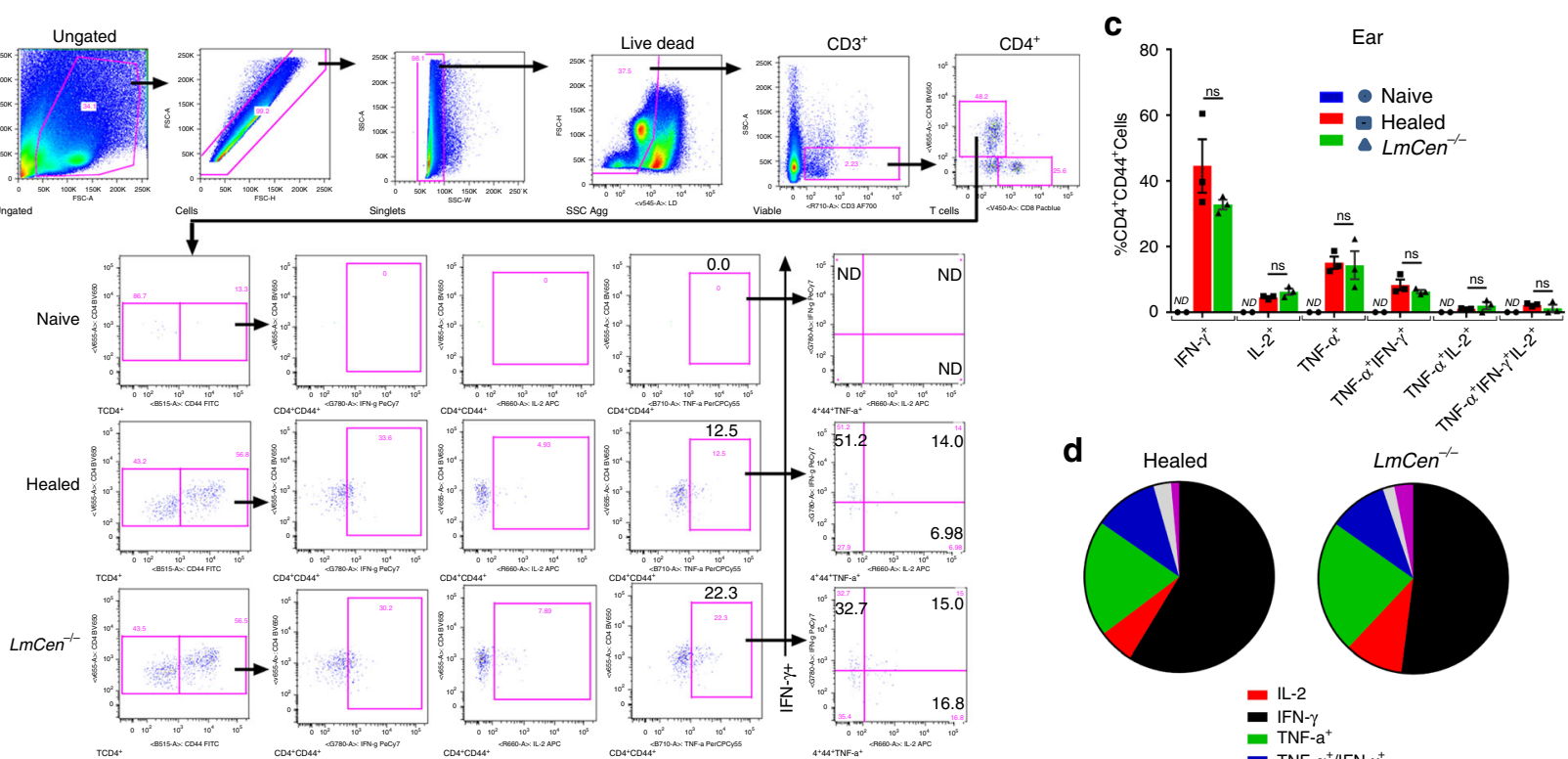

d

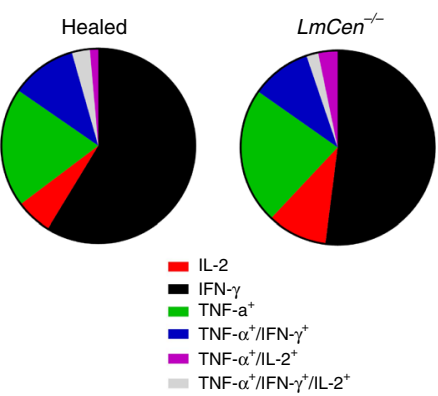

e
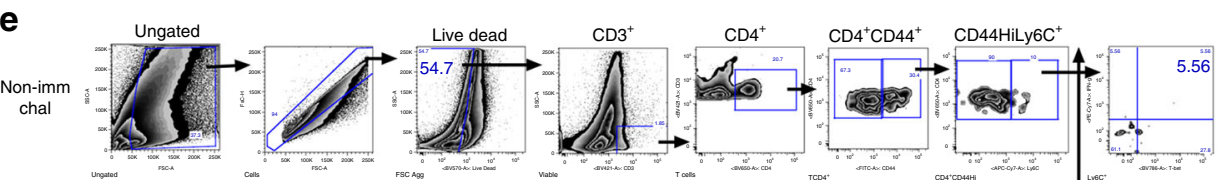

$\mathbf{f}$
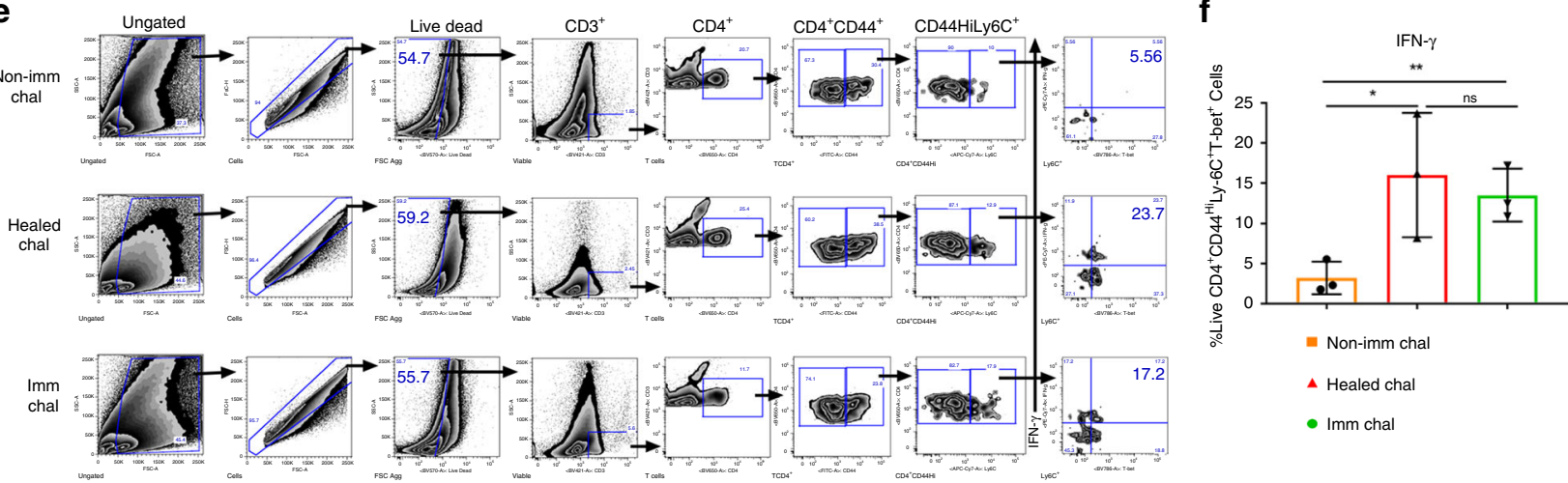

g

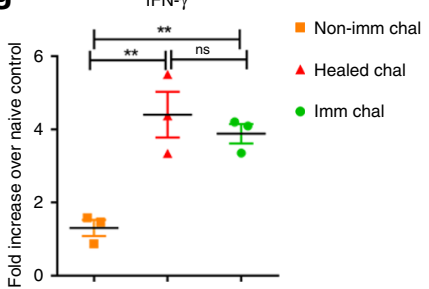

h

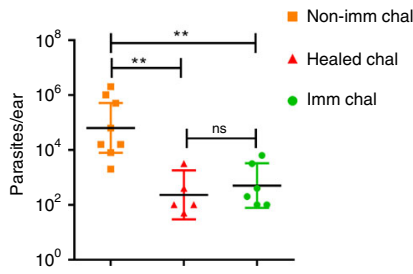

$\Delta$ Healed chal

- Imm chal

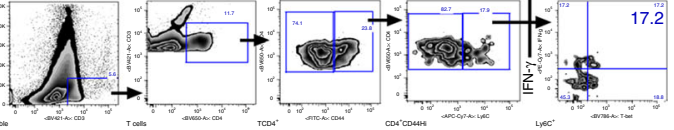

i

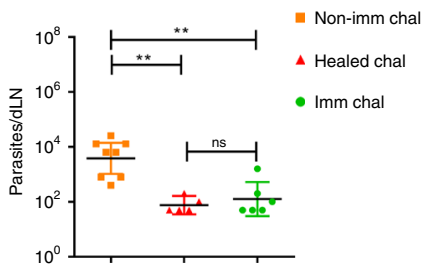

obstacle to using a live vaccine, the risk of disease development, was overcome by engineering a markerless second generation live-attenuated parasite that can confer protection without associated pathology. Live attenuated $\mathrm{LmCen}^{-1-}$ parasites elicited protective immunity in both susceptible $(\mathrm{BALB} / \mathrm{c})$ and resistant $(\mathrm{C} 57 \mathrm{BL} / 6)$ mice and against different strains of $L$. major (WR 2885, FV9 and LV39). Importantly $\mathrm{LmCen}^{-1-}$ parasites elicited protection against sand fly challenge that was deemed necessary but was neither performed or was not demonstrated in previous vaccination studies ${ }^{20,38,41,42}$. These observations using a cutaneous model of infection are consistent with our previous findings that immunization with $\mathrm{LdCen}^{-/-}$parasites were protective against visceral leishmaniasis in different animal models ${ }^{26-28,30,46 .}$ 
Fig. 6 Immunization or leishmanization confer comparable host protection against $\boldsymbol{L}$. major WT infection. a Schematic representation of the experimental approach. b Gating strategies and multiparameter flow-cytometry based analysis for cytokine secreting $T$ cells from pooled ears of naïve, healed and $\mathrm{LmCen}{ }^{-/-}$immunized group of mice. c Multiparameter analysis for multiple cytokine secreting Live $\mathrm{CD} 3^{+} \mathrm{CD} 4^{+} \mathrm{CD} 44^{+} \mathrm{T}$ cells after $20 \mathrm{~h}$ of invitro re-stimulation with freeze-thaw $L$. major antigen $(L m F T A g)$ from pooled ears (2 ears) of naïve control $(n=2)$, healed $(n=3)$ and $L m C e n^{-/-}$ immunized $(n=3)$ group of mice plus naive splenic APCs. Results (mean \pm SEM) are representative of one experiment. Statistical analysis done by Mann-Whitney two-tailed test ( ${ }^{n s} p-0.9$ ). d Pie charts analysis of the cytokine profile of Live $C D 3^{+} \mathrm{CD} 4{ }^{+} \mathrm{CD} 44^{+} \mathrm{T}$ cells. e Gating strategies and zebra plots of immune response in the ear of non-immunized, healed or $\mathrm{LmCen}^{-/-}$immunized mice following needle challenge with wild type $L$. major-parasites. PBS injected naïve mice is control group. $\mathbf{f}$ Ear-derived cells were analyzed and represented as the percentage of IFN- $\gamma$-producing Live CD4 ${ }^{+}$CD44 ${ }^{H i}-$ Ly6C ${ }^{+}$T-bet ${ }^{+}-\mathrm{T}$ cells. Results (mean \pm SEM) are representative of one of two independent experiments with pooled ears (2 ears) samples from healed $(n=6), \mathrm{LmCen}-1-$ immunized $(n=6)$ and age-matched naïve control $(n=6)$ mice. Statistical analysis done by unpaired one-tailed t-test $\left({ }^{n s} p-0.31\right.$, ${ }^{\star} p-0.02$ and $\left.{ }^{\star \star} p-0.005\right)$. $\mathbf{g}$ Ear IFN- $\gamma$ expression were measured by RT-PCR analysis from healed $(n=6), \mathrm{LmCen}^{-1-}$ immunized $(n=6)$ and agematched naïve control $(n=6)$ mice following $20 \mathrm{~h}$ post needle challenge with wildtype L. major-parasites. Results (mean \pm SEM) are representative of one of two independent experiments with pooled ears ( 2 ears) samples ( $n=6$ mice per group). Statistical analysis done by unpaired two-tailed t-test $\left.{ }^{(n s} p-0.48 ;{ }^{\star \star} p<0.009\right)$. Five weeks of post-challenge both ear $\mathbf{h}$ and draining lymph nodes $\mathbf{i}$ parasite load were determined in healed $(n=5), L m C e n^{-/-}$ immunized $(n=6)$ and age-matched naïve control $(n=8)$ mice by serial dilution. Results are represented as geometric means with $95 \% \mathrm{Cl}$ of total 5-8 mice in each group. Data are representative of one experiment. Statistical analysis done by non-parametric Mann-Whitney two-tailed test (ns $p<0.71$; $\left.{ }^{\star \star} p<0.004\right)$.

In previous studies evaluating Leishmania vaccines, researchers have used mice with healed cutaneous lesions following a low dose of wildtype L. major infection as a gold-standard animal model that mimics leishmanization in humans ${ }^{23,38,47}$. In this study, we have also compared $L m C e n^{-1-}$ parasite immunization induced immunity with wildtype $L$. major infected, healed mice (leishmanization). Our results demonstrated comparable immune responses in mice either healed from wildtype infection or immunized with $\mathrm{LmCen}^{-1-}$. It has been shown that chronic parasite infection maintains $\mathrm{Ly} 6 \mathrm{C}^{+} \mathrm{CD} 4{ }^{+}$effector $\mathrm{T}$ cells, and upon challenge with $L m W T$ parasites these are essential for IFN- $\gamma$ production that mediates protection ${ }^{22}$. Our results established that upon challenge with $L m W T$ parasites, both $\mathrm{LmCen}^{-1-}$ immunized and healed mice generated a comparable percentage of $\mathrm{CD}^{+}{ }^{+} \mathrm{Ly} 6 \mathrm{C}^{+} \mathrm{IFN}-\gamma^{+}$effector $\mathrm{T}$ cells. In addition, protection may also be mediated by the circulating effector cells that are recruited by the tissue-resident memory $\mathrm{T}$ cells (Trm) immediately after challenge as was shown in a leishmanization mouse model $^{47}$. Future studies with $L m C e n^{-1-}$ will address the role of Trm cell as well as other memory phenotype T cells in $\mathrm{LmCen}^{-1-}$ vaccine immunity. Moreover, upon $L$. major infected sand fly challenge, both groups are protected, and the levels of protection are comparable in terms of parasite burden. The residual parasite burden observed in both ear and lymph nodes in the $\mathrm{LmCen}^{-1-}$ may be important for maintaining long term protection as was reported in previous studies with leishmanized mice ${ }^{22,48}$. However, unlike leishmanization which involved inoculation of low dose of virulent parasites that caused lesions at the site of injection, immunization with $\mathrm{LmCen}{ }^{-1-}$ parasites is safe as demonstrated by the absence of visible lesions in susceptible and immunodeficient animals post-immunization, in spite of persistence of a low number of $\mathrm{LmCen}^{-1-}$ parasites at the site of inoculation.

In conclusion, this study demonstrated that $\mathrm{LmCen}^{-1-}$ parasites are safe and can protect against a sand fly challenge with a wildtype L. major infection in relevant mouse models. Future studies are required to establish whether vaccination with $\mathrm{LmCen}^{-1-}$ is safe and protective in humans. The combination of old (leishmanization) and new (CRISPR gene editing) technologies can result in major advances in vaccine design that has the potential to protect millions of people from this major neglected disease.

\section{Methods}

Leishmania strain and culture medium. L. major Friedlin (FV9) and L. major LV39 used in this study were routinely passaged into the footpads of BALB/c mice. Amastigotes isolated from infected lesions were grown in M199 medium and promastigotes were cultured at $27^{\circ} \mathrm{C}$ in $\mathrm{M} 199$ medium (pH 7.4) supplemented with $10 \%$ heat-inactivated fetal bovine serum, $40 \mathrm{mM}$ HEPES ( $\mathrm{pH}$ 7.4), $0.1 \mathrm{mM}$ adenine, $5 \mathrm{mgl}^{-1}$ hemin, $1 \mathrm{mgl}^{-1}$ biotin, $1 \mathrm{mgl}^{-1}$ biopterin, $50 \mathrm{U} \mathrm{ml} l^{-1}$ penicillin and $50 \mu \mathrm{g} \mathrm{ml}^{-1}$ streptomycin. Cultures were passaged to fresh medium at a 40 -fold dilution once a week. The growth curve of $L$. major promastigotes was obtained by inoculating the parasite at $1 \times 10^{6} / \mathrm{ml}$ into the 96 well plate $(150 \mu \mathrm{l} /$ well $)$ in quadruplicate, the $\mathrm{OD}$ values were measured once a day for 4 days.

L. major WR 2885 strain was used to infect sand flies and grown at $27^{\circ} \mathrm{C}$ in Schneider's medium supplemented with $10 \%$ heat-inactivated FCS, penicillin $(100$ $\mathrm{U} / \mathrm{ml})$, streptomycin $(100 \mu \mathrm{g} / \mathrm{ml}), 2 \mathrm{mM}$ l-glutamine.

The WR2885 strain was isolated in August 29, 2008 from a lesion on the right upper arm of a US soldier at the Walter Reed hospital, Bethesda, MD. It was acquired in Iraq and the cloned line was identified as L. major by polymerase chain reaction (PCR) and by isozyme assessment by a College of American Pathologists certified laboratory. The WR2885 strain is shown to have superior colonization and transmissibility by sand flies to mice resulting in more severe pathology (larger lesion size and higher parasite loads $)^{38,49}$.

CRISPR plasmid construction. The pLdCNLm221410a\&b plasmid vector was generated as follows: 1) A 276 bp PCR fragment containing gRNALm221410a, hepatitis delta virus and hammerhead ribozymes and gRNALm221410b guide coding sequences was amplified with primers Lm221410a and Ld221410b from the gRNA $241510+$ MT co-expression vector previously described 33,342 ). The PCR product from step 1 was digested with Bbs I and inserted into the Bbs I digested pLdCN vector ${ }^{33,34}$ to generate the pLdCNLm221410a\&b plasmid vector which was verified by sequencing analysis at the McGill University and Genome Quebec Innovation Center.

Guide RNA sequences and the oligonucleotide donor used in this study are listed below and their locations in the centrin gene (LmjF.22.1410) locus are indicated in the Supplementary Fig. 1.

Lm221410a (containing gRNAa guide coding sequence): 5'ATCGAAGACCT TTGTCTTCTCGCAATCCTTCTGCTGTTTTAGAGCTAGAAATAGCAAG Lm221410b (containing gRNAb guide coding sequence): 5'ATCGAAGACC CAAACTTGAGAGGGAAAGCAACGGACACCATGACGAGCTTACTC Oligo donor (Lm221410): 5'ATTTCGTGCTTCTCGCAATCCTTCTCAACGG ATGATAGTGCG CGTGTGCG

Lm221410F1: 5' CCTTTGCGAACTTGAAGGAG

Lm221410R1: 5' ACGGACAGCACAACCATACA

Lm221410F2: 5' CTAGGCGATCGAGTCTTTGG

Lm221410R2: 5' ATCGTGATTCGCTTGTTTCC

Selection of centrin gene deleted clones and single-cell cloning. Leishmania transfections were performed as previously described ${ }^{37}$. Briefly, $10 \mu \mathrm{g}$ pLdCNLm221410a\&b plasmid DNA was electroporated into $1 \times 10^{8}$ early stationary phase $L$. major promastigotes. The transfected cells were then selected with $\mathrm{G} 418(100 \mu \mathrm{g} / \mathrm{ml})$ for 2 weeks. Once the transfected L. major culture was established, the surviving promastigotes were subjected to three rounds of transfection with the oligonucleotide donor ( $\mathrm{Lm} 221410$ oligo donor); $10 \mu \mathrm{l} 100 \mu \mathrm{M}$ singlestrand oligonucleotide donor was used per transfection, once every three days. After the third oligonucleotide donor transfection, the Leishmania promastigotes were counted and inoculated into 96 well plates at one promastigote per $100 \mu \mathrm{l}$ medium per well. The growth of Leishmania cells in 96 well plates was monitored under microscope. After culture for three weeks in 96 well plates, parasites from the relatively slow-growing clones were expanded in 24 well plates. The slow-growing clones were selected since this represents the phenotype for loss of the centrin 
gene $^{35}$. The genomic DNA extracted from the slow growth clones were subjected to PCR and DNA sequencing analysis to confirm deletion of the centrin gene.

To remove the pLdCNLm221410a\&b plasmid, the centrin gene deleted $L$. major strain was grown in G418 free medium for several weeks, individual clones were then grown in duplicate plates where one plate contained media without G418 and the duplicate plate contained media with G418. Clones that had lost the plasmid were identified since they lost the ability to survive in the presence of G418.

Genome sequence analysis of $\mathbf{L m C e n}{ }^{-/-}$. Complete genome sequencing of two clones from $\mathrm{LmCen}^{-1-}$ was determined by MiSeq genome sequencing reaction on an Illumina sequencing instrument at the sequencing core facility at the Center for Biologics Evaluation and Research. $\mathrm{LmCen}{ }^{-1-}$ sequence reads were aligned against Leishmania major Friedlin strain reference genome (retrieved from www.tritrypdb. org) using the Burrows-Wheeler Aligner Maximal Exact Match algorithm (BWAMEM $)^{50}$. The alignments were converted to BED files using samtools and processed using the bedtools software package ${ }^{51,52}$. The bedtools coverage command was used with the "-d" option in conjunction with the genomic intervals containing the centrin genes to count the read depth at each position in the coverage of centrin genes shown in Fig. 2a with a 200 bp window. The bedtools coverage command was used in conjunction with gene coordinates extracted from the gff genomic annotation file (retrieved from www.tritrypdb.org ${ }^{53}$ ) to compute the percent coverage of each gene as shown in Fig. 2c. Genes with less than 100 percent coverage were manually inspected for a sharp drop-off in coverage (deletion) versus a gradual decline in close proximity to an inverse increase in coverage in a tandem gene (misalignment).

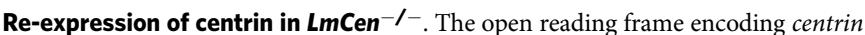
gene was cloned into the SpeI sites of the Leishmania expression plasmid pKSNeo. $\mathrm{LmCen}^{-1-}$ parasites were transfected with the plasmid and recombinant parasites were selected using $50 \mathrm{\mu g} / \mathrm{ml} \mathrm{G} 418$ to obtain $\mathrm{LmCen}^{-1-}$ parasites re-expressing centrin gene termed $\mathrm{LmCen}^{-1-}$ Addback $\left(\mathrm{LmCen}^{-1-} \mathrm{AB}\right)$.

Southern hybridization. Total genomic DNA was isolated from promastigotes with the Wizard genomic DNA purification kit (Promega Biosciences). The DNA $(5 \mu \mathrm{g})$ was digested with restriction enzyme BglII and the digestion products were separated on $1 \%$ agarose gels and transferred to positively charged nitrocellulose membranes. Southern blot analysis of the resolved DNA was performed as described previously using a ${ }^{32} \mathrm{p}$-labeled $L$. major centrin ORF nucleotide sequence as a probe $\mathrm{e}^{39}$. The DNA fragments were ligated into PCR2.1-Topo vector and the nucleotide sequence of the probe was determined to ensure fidelity. The plasmid containing the correct probe was digested with EcoRI, gel purified and labeled with Random Prime it-II kit using ${ }^{32} \mathrm{p}$-dCTP (Agilent Technologies).

Mice infection and immunization. Female 6- to 8-wk-old C57BL/6 and $\mathrm{BALB} / \mathrm{c}$ mice were immunized and/or infected with $1 \times 10^{6}$ total stationary phase $L m C e n^{-1-}$ or L. major wildtype ( $L m W T$ ) parasites by intradermal injection in the left ear in $10 \mu \mathrm{l}$ PBS. For challenge infections, age-matched naive and seven-week post immunized mice (both $\mathrm{C} 57 \mathrm{BL} / 6$ and $\mathrm{BALB} / \mathrm{c}$ ) were challenged in the right ear with 750 metacyclic $L$. major (WR 2885) wildtype promastigotes intradermally. The numbers of $L$. major (WR 2885) parasites in the infectious inoculum were determined by a titration analysis revealing that 750 metacyclic parasites cause reproducible pathology in $\mathrm{BALB} / \mathrm{c}$ mice ear. For leishmanization, mice were infected with $1 \times 10^{4}$ metacyclic promastigotes of $L$. major Friedlin (FV9) strain by intradermal needle injection in the ear. After 12 weeks of post-infection, healed mice were challenged on the contralateral ear with $1 \times 10^{5}$ metacyclic $L$. major (FV9) wildtype ( $L m W T)$ parasites by needle inoculation.

Lesion size was monitored up to 10 weeks post-challenge by measuring the diameter of the ear lesion using a direct reading Vernier caliper. Parasite burden in the challenged ear and draining lymph node (dLN) was estimated by limiting dilution analysis as previously described ${ }^{37}$. Briefly, two sheets of ear dermis were separated, deposited in DMEM containing $100 \mathrm{U} / \mathrm{ml}$ penicillin, $100 \mu \mathrm{g} / \mathrm{ml}$ streptomycin, and $0.2 \mathrm{mg} / \mathrm{ml}$ Liberase CI purified enzyme blend (Roche Diagnostics Corp.), and incubated for $1-2 \mathrm{~h}$ at $37^{\circ} \mathrm{C}$. Digested tissue was processed in a tissue homogenizer (Medimachine; Becton Dickinson) and filtered through a $70 \mu \mathrm{m}$ cell strainer (Falcon Products). Parasite titrations in the ear and dLN were performed by serial dilution (1:1 dilutions) of tissue homogenates in 96-well flatbottom microtiter plates (Corning, Corning, NY) in M199 cell culture media in duplicate and incubated at $26^{\circ} \mathrm{C}$ without $\mathrm{CO}_{2}$ for $7-10$ days. The greatest dilution yielding viable parasites was recorded and data are presented the mean parasite dilution \pm SEM. For histology, challenged ears were fixed, after 10 weeks of post WT parasite infection, in fixative solutions (10\% buffered formalin phosphate solution) and paraffin-embedded sections were stained with hematoxylin and eosin (H\&E) (Histoserv Inc.). The stained slides were visualized and photographed using KEYENCE digital microscope.

BALB/c mice were immunized subcutaneously in the footpad with $2 \times 10^{8}$ $\mathrm{LmCen}{ }^{-1-}$ parasites of the Friedlin strain or injected with PBS. After 6 weeks both groups were challenged with $10^{4}$ virulent metacyclics of LV39 L. major parasites intra-dermally in the ear. Ear lesions of vaccinated and non-vaccinated mice (PBS group) challenged with L. major LV39 metacyclic promastigotes were measured at least once a week from week 1 post challenge to week 10 post challenge.

6-8 weeks old female BALB/c, IFN- $\gamma$ KO (C57BL/6 background) and Rag2 KO (C57BL/6 J (B6(Cg)-Rag2tm1.1Cgn/J) mice were subcutaneously inoculated with $1 \times 10^{7}$ of $L m W T$ (Friedlin V9) or $L m C e n^{-l-}$ into the right hind footpad. Following infection, footpad swelling was measured weekly by digital caliper. Parasite burden in infected footpad was measured at 5 weeks after infection in $\mathrm{BALB} / \mathrm{c}$, at or at 15 weeks in IFN- $\gamma \mathrm{KO}$ and Rag2 KO mice. 6-8 old weeks female STAT-1 KO (BALB/c background) mice were injected subcutaneously in the footpad with $2 \times 10^{8} \mathrm{LmCen}^{-1-}$ parasites of the Friedlin strain or infected with $2 \times$ $10^{8}$ L. major WT parasites of the Friedlin strain. Footpad swelling of both groups was measured at least once a week from week 1 after injection to week 7. After 7 weeks both groups were sacrificed, and parasite burden was determined. Footpad lesions was excised and then homogenized with a cell strainer in $3 \mathrm{ml}$ of Schneider's Drosophila medium (Gibco, US) supplemented with 20\% heatinactivated fetal calf serum and Penicillin-Streptomycin (0.1\%).

\section{Sand fly infection and transmission of $\boldsymbol{L}$. major to immunize mice. Female} Lutzomyia longipalpis (Jacobina strain, reared at the Laboratory of Malaria and Vector Research, NIAID) sand flies (5-7 days old) were infected by artificial feeding through a chick skin membrane on a suspension of $5 \times 10^{6} \mathrm{~L}$. major (WR 2855) procyclic promastigotes $/ \mathrm{ml}$ of heparinized defibrinated blood containing penicillin and streptomycin. Flies with mature infections were used for transmission $^{54}$. One day before transmission the sucrose diet was removed. Mice were anesthetized by intraperitoneal injection of $30 \mu \mathrm{l}$ of ketamine/xylazine $(100 \mathrm{mg} / \mathrm{ml})$. Ointment was applied to the eyes to prevent corneal dryness. Ten infected flies were applied to right ears of both $\mathrm{LmCen}^{-/-}$immunized and age-matched naïve C57BL/6 mice through a meshed surface of vials which were held in place by custom made clamps. The flies were allowed to feed on the exposed ear for a period of $2-3 \mathrm{~h}$ in the dark at $23^{\circ} \mathrm{C}$ and $50 \%$ humidity. Following exposure, the number of flies per vial with or without a blood meal was counted to determine the influence of feeding intensity on transmission frequency. Animals were sacrificed 10 weeks post sand fly exposure \& organ parasite burden were determined by serial dilution as described above.

Human macrophage infection. Human elutriated monocytes were obtained from NIH blood bank from healthy US blood donors. Only monocytes that tested CMV negative were used in this study. Monocytes were re-suspended at $2 \times 10^{5} \mathrm{cells} / \mathrm{ml}$ in RPMI medium containing $10 \%$ FBS and human macrophage colony-stimulating factor $(20 \mathrm{ng} / \mathrm{ml}$, ProSpec), plated in a volume of $0.5 \mathrm{ml}$ in eight-chamber Lab-Tek tissue culture slides (Miles Laboratories) and incubated for 7 days for differentiation into macrophages. The differentiated macrophages were infected with stationary phase $\mathrm{LmWT}$ or $\mathrm{LmCen} \mathrm{C}^{-1-}$ promastigotes (10:1 parasite-to-macrophage ratio). After incubation for $6 \mathrm{~h}$ at $37^{\circ} \mathrm{C}$ in $5 \% \mathrm{CO}_{2}$, the free extracellular parasites were removed by RPMI washes and the cultures were incubated in macrophage culture medium for an additional $24 \mathrm{~h}$. The culture medium was removed, and macrophages infected with $L m W T$ or $L m C e n^{-1-}$ were stained with Diff-Quik staining reagent. Percentages of infected macrophages were determined by counting a minimum of 100 macrophages per sample under the microscope. Results are shown as mean \pm SEM for three independent counts for each infection on days $1-8$.

RT-PCR. Total RNA was extracted from the ears tissue using a Pure Link RNA Mini kit (Ambion). Total RNA (400 ng) was reverse transcribed into cDNA using random hexamers with a high-capacity cDNA reverse transcription kit (Applied Biosytems). Gene expressions were determined using TaqMan Gene Expression Master Mix and premade TaqMan Gene Expression assays (Applied Biosystems) using a CFX96 Touch real-time system (Bio-Rad, CA) and the data were analyzed with CFX Manager software. The TaqMan Gene Expression Assay ID (Applied Biosystems) of IFN- $\gamma$ (Mm01168134_m1) and GAPDH (Mm99999915_g1). Expression values were determined by the $2^{-\Delta \Delta \mathrm{Ct}}$ method where samples were normalized to GAPDH expression and determined relative to naive sample.

Measurement of cytokine expression from ear-derived $\mathrm{CD4}^{+} \mathrm{T}$ cell populations by flow cytometry. To determine the comparative immune response at preor $20 \mathrm{~h}$ post-L. major WT needle challenge, single-cell suspensions from ear of healed (leishmanized) and $\mathrm{LmCen}^{-1-}$ immunized mice were incubated with $1 \times$ $10^{6}$ T-cell depleted (Miltenyi Biotech) naïve spleen cells (APCs), with $50 \mu \mathrm{g} / \mathrm{ml}$ freeze-thaw $L$. major antigen ( $L m F T A g)$ in flat bottom 48 -well plates at $37^{\circ} \mathrm{C}$ for 12-14 h. During last $4 \mathrm{~h}$ of culture, protein Transport Inhibitor (BD Golgiplug, BD BioSciences) was added to the wells. Cells were then blocked at $4{ }^{\circ} \mathrm{C}$ with rat $\alpha$ mouse CD16/32 $(5 \mu \mathrm{g} / \mathrm{ml})$ from BD BioSciences for $20 \mathrm{~min}$. For surface staining, cells were then stained with $\alpha$-mouse CD3 AF-700 (BD BioSciences), $\alpha$-mouse CD4 BV-650 (Biolegend) and $\alpha$-mouse CD44 FITC (BD BioSciences) or $\alpha$-mouse CD3 BV421 (BD BioSciences), $\alpha$-mouse CD4 BV-650 (Biolegend), $\alpha$-mouse Ly-6C APC-Cy7 (BD BioSciences) and $\alpha$-mouse CD44 FITC (BD BioSciences) for $30 \mathrm{~min}$ (each with $1 / 300$ dilution; $4{ }^{\circ} \mathrm{C}$ ). The cells were then stained with LIVE/DEAD fixable aqua (Invitrogen/Molecular Probes) to stain dead cells. Cells were washed with wash buffer and fixed with the Cytofix/Cytoperm Kit (BD Biosciences) for 20 
min (room temperature). Intracellular staining was done with $a$-mouse IL-2 APC (BD BioSciences), $\alpha$-mouse IFN- $\gamma$ PE-Cy7 (Biolegend) and $\alpha$-mouse TNF- $\alpha$ PerCP-Cy5.5 (Biolegend), for 30 min (each with 1:300 dilution; $4^{\circ} \mathrm{C}$ ). In some experiments samples were treated with Foxp3 Fixation/Permeabilization Buffer (ebioscience) and then stained with a-mouse T-bet -BV786 (BD Biosciences) according to manufacturer's instruction. Cells were acquired on Symphony (BD Biosciences) analyzer equipped with 350, 405, 445, 488, 561, 638 and $785 \mathrm{~nm}$ LASER lines using DIVA software (v8). Data were analyzed with the FlowJo software version 9.9.6 (BD, San Jose CA). For analysis, first doublets were removed using width parameter; dead cells were excluded based on staining with the Live/ Dead Aqua dye. Lymphocytes were identified according to their light-scattering properties. $\mathrm{CD} 4^{+} \mathrm{T}$-cells were identified as $\mathrm{CD} 3^{+}$lymphocytes uniquely expressing CD4. Upon further gating intracellular cytokines were measured in Live CD4 ${ }^{+}$ $\mathrm{CD} 44^{\mathrm{Hi}}{ }_{\mathrm{Ly} 6 \mathrm{C}^{+}} \mathrm{T}$-bet ${ }^{+}$cells. Fluorescence minus one control was used for proper gating of positive events for designated cytokines (Supplementary Fig. 4C, D)

Immunosuppression by dexamethasone injection. To determine the safety of Centrin deficient $\mathrm{LmCen}^{-1-}$ parasites in immune-suppressive condition, 4-6 weeks old BALB/c mice were divided into three groups. Group-1 $(n=6)$ were infected with $1 \times 10^{6}$ stationery phase $L m W T$ parasites and Group-2 $(n=6)$ and Group-3 $(n=12)$ animals were immunized with $1 \times 10^{6}$ stationery phase $\mathrm{LmCen}^{-1-}$ parasites in a $10 \mu \mathrm{l}$ volume of PBS through intradermal (into the ear dermis) routes. After 10 weeks of post infection, only Group-3 animals were treated with $2 \mathrm{mg} / \mathrm{kg}$ Dexamethasone sodium phosphate (Sigma Aldrich) in PBS by subcutaneous injection three times for one week. Four weeks after this treatment (total 15 weeks post infection); all the groups were sacrificed and evaluated for parasite burden by serial dilution as described above. Development of pathology \& lesion size in the ear was assessed at 15 weeks post infection by measuring the diameter of the lesion.

Characterization of centrin deleted parasites isolated from $\mathrm{LmCen}^{-1-}$ plus DXM-treated group was done by Polymerase chain reaction. Total Genomic DNA was isolated from the parasites recovered from $L m W T$ and $\mathrm{LmCen}^{-1-}$ plus DXMtreated group according to the manufacturer information (DNeasy Blood \& Tissue Kit, Qiagen). PCR was performed with $L$. major centrin gene-specific primer (For5'-ATGGCTGCGCTGACGGATGAACAGATTCGC-3'; Rev-5'-CTTTCCACGC ATCTGCAGCATCACGC- $3^{\prime}$ ) which target the amplification of the 450-bp. A reaction mixture was prepared containing $10 \times$ Buffer (Invitrogen), $0.2 \mathrm{mmol} / \mathrm{l}$ each deoxyribonucleotide (Invitrogen), $1 \mu \mathrm{mol} / \mathrm{l}$ each primer, 1.25 units of Taq polymerase (Invitrogen) and $200 \mathrm{ng}$ of DNA samples in a final volume of $50 \mu \mathrm{l}$. The PCR conditions were as follows: denaturation at $94^{\circ} \mathrm{C}$ for $3 \mathrm{~min}$, followed by 35 cycles of $94^{\circ} \mathrm{C}$ for $20 \mathrm{~s}, 58^{\circ} \mathrm{C}$ for $20 \mathrm{~s}$ and $68^{\circ} \mathrm{C}$ for $35 \mathrm{~s}$ with a final extension of $68^{\circ} \mathrm{C}$ for $5 \mathrm{~min}$. The amplification reactions were analyzed by $1 \%$ agarose gel electrophoresis, followed by ethidium bromide staining and visualization under UV light. DNA from the reference plasmid (PCR 2.1 TOPO) containing centrin gene was used as a positive control.

Statistical analysis. Statistical analysis of differences between means of groups was determined Student's $t$ test using Graph Pad Prism 7.0 software. The statistical tests and the significance values are described in the figure legends.

Ethical statement. The animal protocol for this study has been approved by the Institutional Animal Care and Use Committee at the Center for Biologics Evaluation and Research, US FDA (ASP 1995\#26). The animal protocol is in full accordance with "The guide for the care and use of animals as described in the US Public Health Service policy on Humane Care and Use of Laboratory Animals 2015". The use of blood components (elutriated monocytes) from the Department of Transfusion Medicine, NIH was approved by the institutional Research Involving Human Subjects Committee (RIHSC\#03-120B) at the US FDA. All animal studies at Ohio State University were performed in accordance with NIH guidelines for the humane care and use of animals and were approved by OSU IACUC. Animal experimental procedures performed at the National Institute of Allergy and Infectious Diseases (NIAID) were reviewed by the NIAID Animal Care and Use Committee under animal protocol LMVR4E. The NIAID DIR Animal Care and Use Program complies with the Guide for the Care and Use of Laboratory Animals and with the NIH Office of Animal Care and Use and Animal Research Advisory Committee guidelines. Detailed NIH Animal Research Guidelines can be accessed at https://oma1.od.nih.gov/manualchapters/intramural/3040-2/. Animal experimental procedures performed at Nagasaki University were approved by the Institutional Animal Research Committee of Nagasaki University (No.1606211317 and 1505181227), the Nagasaki University Recombinant DNA Experiments Safety Committee (No. 1403041262 and 1407221278), and performed according to Japanese law for the Humane Treatment and Management of Animals.

Reporting summary. Further information on research design is available in the Nature Research Reporting Summary linked to this article.

\section{Data availability}

All relevant data are available in the main text and supplementary information, are available upon reasonable requests to the authors. Source data are provided with this paper.

Received: 17 January 2019; Accepted: 13 June 2020;

Published online: 10 July 2020

\section{References}

1. Burza, S., Croft, S. L. \& Boelaert, M. Leishmaniasis. Lancet 392, 951-970 (2018)

2. Alvar, J. et al. Leishmaniasis worldwide and global estimates of its incidence PLoS One 7, e35671 (2012).

3. WHO. Leishmaniasis in high-burden countries: an epidemiological update based on data reported in 2014. Relev. Epidemiol. Hebd., https://doi.org/ 10.1186/1750-9378-2-15.Voir (2016).

4. McCall, L. I., Zhang, W. W. \& Matlashewski, G. Determinants for the development of visceral leishmaniasis disease. PLoS Pathogens 9, e1003053 (2013)

5. Matlashewski, G. et al. Research priorities for elimination of visceral leishmaniasis. The Lancet Global Health, https://doi.org/10.1016/S2214-109X (14)70318-3 (2014).

6. Gillespie, P. M. et al. Status of vaccine research and development of vaccines for leishmaniasis. Vaccine 34, 2992-2995 (2016).

7. Alvar, J. et al. Case study for a vaccine against leishmaniasis. Vaccine, https:// doi.org/10.1016/j.vaccine.2012.11.080 (2013).

8. Selvapandiyan, A. et al. Generation of growth arrested Leishmania amastigotes: A tool to develop live attenuated vaccine candidates against visceral leishmaniasis. Vaccine, https://doi.org/10.1016/j.vaccine.2014.05.009 (2014).

9. Row, R. The curative value of Leishmania culture "vaccine" in oriental sore. Br. Med. J. 1, 540 (1912).

10. Marzinowsky, E. I. Oriental sore and immunity against it. Trans. R. Soc. Trop. Med. Hyg., https://doi.org/10.1016/S0035-9203(24)90754-1 (1924).

11. Khamesipour, A. et al. Leishmanization: Use of an old method for evaluation of candidate vaccines against leishmaniasis. Vaccine, https://doi.org/10.1007/ BF02809767 (2005)

12. Bogdan, C. Mechanisms and consequences of persistence of intracellular pathogens: leishmaniasis as an example. Cell. Microbiol., https://doi.org/ 10.1111/j.1462-5822.2008.01146.x (2008)

13. Maspi, N., Abdoli, A. \& Ghaffarifar, F. Pro- and anti-inflammatory cytokines in cutaneous leishmaniasis: a review. Pathog. Global Health, https://doi.org/ 10.1080/20477724.2016.1232042 (2016).

14. Hurdayal, R. \& Brombacher, F. The role of IL-4 and IL-13 in cutaneous leishmaniasis. Immunol. Lett., https://doi.org/10.1016/j.imlet.2013.12.022 (2014).

15. Kaye, P. \& Scott, P. Leishmaniasis: complexity at the host-pathogen interface. Nat. Rev. Microbiol., https://doi.org/10.1038/nrmicro2608 (2011).

16. Coler, R. N. et al. Immunization with a polyprotein vaccine consisting of the $\mathrm{T}$-cell antigens thiol-specific antioxidant, Leishmania major stress-inducible protein 1, and Leishmania elongation initiation factor protects against leishmaniasis. Infect. Immun., https://doi.org/10.1128/IAI.70.8.42154225.2002 (2002).

17. Jajarmi, V. et al. Immunization against Leishmania major infection in BALB/c mice using a subunit-based DNA vaccine derived from TSA, LmSTI1, KMP11, and LACK predominant antigens. Iran. J. Basic Med. Sci. 22, 1493-1501 (2019).

18. Gonzalo, R. M. et al. A heterologous prime-boost regime using DNA and recombinant vaccinia virus expressing the Leishmania infantum P36/LACK antigen protects BALB/c mice from cutaneous leishmaniasis. Vaccine, https:// doi.org/10.1016/S0264-410X(01)00427-3 (2002).

19. Zaph, C., Uzonna, J., Beverley, S. M. \& Scott, P. Central memory T cells mediate long-term immunity to Leishmania major in the absence of persistent parasites. Nat. Med., https://doi.org/10.1038/nm1108 (2004).

20. Peters, N. C. et al. Vector transmission of Leishmania abrogates vaccineinduced protective immunity. PLoS Pathog., https://doi.org/10.1371/journal. ppat.1000484 (2009).

21. Darrah, P. A. et al. Multifunctional TH1 cells define a correlate of vaccinemediated protection against Leishmania major. Nat. Med., https://doi.org/ 10.1038/nm1592 (2007).

22. Peters, N. C. et al. Chronic parasitic infection maintains high frequencies of short-lived Ly6C+CD4+ effector $\mathrm{T}$ cells that are required for protection against re-infection. PLoS Pathog., https://doi.org/10.1371/journal. ppat.1004538 (2014)

23. Hohman, L. S. \& Peters, N. C. CD4+ T cell-mediated immunity against the phagosomal pathogen leishmania: implications for vaccination. Trends Parasitol., https://doi.org/10.1016/j.pt.2019.04.002 (2019). 
24. Selvapandiyan, A. et al. Centrin gene disruption impairs stage-specific basal body duplication and cell cycle progression in Leishmania. J. Biol. Chem., https://doi.org/10.1074/jbc.M402794200 (2004).

25. Selvapandiyan, A. et al. Centrin1 is required for organelle segregation and cytokinesis in Trypanosoma brucei. Mol. Biol. Cell, https://doi.org/10.1091/ mbc.E07-01-0022 (2007).

26. Selvapandiyan, A. et al. Intracellular replication-deficient Leishmania donovani induces long lasting protective immunity against visceral leishmaniasis. J. Immunol., https://doi.org/10.4049/jimmunol.0900276 (2009).

27. Fiuza, J. A. et al. Induction of immunogenicity by live attenuated Leishmania donovani centrin deleted parasites in dogs. Vaccine, https://doi.org/10.1016/j. vaccine.2013.01.048 (2013)

28. Dey, R. et al. Characterization of cross-protection by genetically modified liveattenuated Leishmania donovani parasites against Leishmania mexicana. J. Immunol., https://doi.org/10.4049/jimmunol.1303145 (2014).

29. Gannavaram, S. et al. Methods to evaluate the preclinical safety and immunogenicity of genetically modified live-attenuated Leishmania parasite vaccines. in Methods in Molecular Biology., https://doi.org/10.1007/978-14939-3387-7_35 (2016).

30. Banerjee, A. et al. Live attenuated Leishmania donovani centrin gene-deleted parasites induce IL-23-dependent IL-17-protective immune response against visceral leishmaniasis in a murine model. J. Immunol., https://doi.org/10.4049/ jimmunol.1700674 (2017).

31. Fiuza, J. A. et al. Vaccination using live attenuated Leishmania donovani centrin deleted parasites induces protection in dogs against Leishmania infantum. Vaccine, https://doi.org/10.1016/j.vaccine.2014.11.039 (2015).

32. Zhang, W. W. \& Matlashewski, G. CRISPR-Cas9-mediated genome editing in Leishmania donovani. MBio 6, e00861-15 (2015).

33. Zhang, W.-W., Lypaczewski, P. \& Matlashewski, G. Optimized CRISPR-Cas9 genome editing for Leishmania and its use to target a multigene family, induce chromosomal translocation, and study DNA break repair mechanisms. mSphere, https://doi.org/10.1128/mSphere.00340-16 (2017).

34. Zhang, W.-W., Lypaczewski, P. \& Matlashewski, G. Application of CRISPRCas9 mediated genome editing in Leishmania. in Methods in Molecular Biology (eds. Ginger, M., Zilberstein, D. \& Michels, P.) (Springer Nature, 2019).

35. Selvapandiyan, A. et al. Expression of a mutant form of Leishmania donovani centrin reduces the growth of the parasite. J. Biol. Chem., https://doi.org/ 10.1074/jbc.M106806200 (2001).

36. Zhang, W. W., Charest, H. \& Matlashewski, G. The expression of biologically active human p53 in Leishmania cells: a novel eukaryotic system to produce recombinant proteins. Nucleic Acids Res., https://doi.org/10.1093/nar/ 23.20.4073 (1995).

37. Zhang, W. W. \& Matlashewski, G. Screening Leishmania donovani-specific genes required for visceral infection. Mol. Microbiol. 77, 505-517 (2010).

38. Peters, N. C. et al. Evaluation of recombinant Leishmania polyprotein plus glucopyranosyl lipid A stable emulsion vaccines against sand fly-transmitted Leishmania major in C57BL/6 mice. J. Immunol., https://doi.org/10.4049/ jimmunol.1201676 (2012).

39. Gannavaram, S. et al. Whole genome sequencing of live attenuated Leishmania donovani parasites reveals novel biomarkers of attenuation and enables product characterization. Sci. Rep. 7, 1-10 (2017).

40. Medeiros, L. C. S. et al. Rapid, selection-free, high-efficiency genome editing in protozoan parasites using CRISPR-cas9 ribonucleoproteins. Mbio, https://doi. org/10.1128/mBio.01788-17 (2017).

41. Gomes, R. et al. KSAC, a defined Leishmania antigen, plus adjuvant protects against the virulence of $\mathrm{L}$. major transmitted by its natural vector Phlebotomus duboscqi. PLoS Negl. Trop. Dis., https://doi.org/10.1371/journal. pntd.0001610 (2012).

42. Rogers, M. E., Sizova, O. V., Ferguson, M. A. J., Nikolaev, A. V. \& Bates, P. A. Synthetic glycovaccine protects against the bite of Leishmania- infected sand flies. J. Infect. Dis., https://doi.org/10.1086/505584 (2006).

43. Titus, R. \& Ribeiro, J. Salivary gland lysates from the sand fly Lutzomyia longipalpis enhance Leishmania infectivity. Science. 239, 1306-1308 (1988).

44. Rogers, M. E., Ilg, T., Nikolaev, A. V., Ferguson, M. A. J. \& Bates, P. A. Transmission of cutaneous leishmaniasis by sand flies is enhanced by regurgitation of fPPG. Nature 430, 463-467 (2004).

45. Peters, N. C. et al. In vivo imaging reveals an essential role for neutrophils in leishmaniasis transmitted by sand flies. Science. 321, 970-974 (2008).

46. Fiuza, J. A. et al. Intradermal immunization of Leishmania donovani centrin knock-out parasites in combination with salivary protein LJM19 from sand fly vector induces a durable protective immune response in hamsters. PLoS Negl. Trop. Dis., https://doi.org/10.1371/journal.pntd.0004322 (2016).

47. Glennie, N. D. et al. Skin-resident memory CD4+ T cells enhance protection against Leishmania major infection. J. Exp. Med. 212, 1405-1414 (2015).
48. Scott, P. \& Novais, F. O. Cutaneous leishmaniasis: immune responses in protection and pathogenesis. Nat. Rev. Immunol. 16, 581-592 (2016).

49. Gomes, R. et al. Immunity to sand fly salivary protein LJM11 modulates host response to vector-transmitted Leishmania conferring ulcer-free protection. J. Invest. Dermatol. 132, 2735-2743 (2012).

50. Li, H. Aligning sequence reads, clone sequences and assembly contigs with BWA-MEM. https://arxiv.org/abs/1303.3997 (2013).

51. Li, H. et al. The sequence alignment/map format and SAMtools. Bioinformatics 25, 2078-2079 (2009).

52. Quinlan, A. R. \& Hall, I. M. BEDTools: a flexible suite of utilities for comparing genomic features. Bioinformatics 26, 841-842 (2010).

53. Aslett, M., Aurrecoechea, C., Berriman, M. \& Al., E. TriTrypDB: a functional genomic resource for the Trypanosomatidae. Nucleic Acids Res. 38, D457-D462 (2010).

54. Kamhawi, S., Belkaid, Y., Modi, G., Rowton, E. \& Sacks, D. Protection against cutaneous leishmaniasis resulting from bites of uninfected sand flies. Science, https://doi.org/10.1126/science.290.5495.1351 (2000).

\section{Acknowledgements}

Funding was provided from the Global Health Innovative Technology Fund, the Canadian Institutes of Health Research (to GM), intramural funding from CBER, FDA (to H.L.N.), and the Fonds de recherche du Québec - Santé (to P.L.). The findings of this study are an informal communication and represent the authors' own best judgments. These comments do not bind or obligate the Food and Drug Administration. This research is supported, in part, by the Intramural Reserch Programm of the NIH, National Institute of Allergy and Infectious Diseases, (F.O., I.V.C.A., T.D.V., C.M., J.O., T.D., S.K., J.G.V.).

\section{Author contributions}

W.W.Z., P.L., S.G., R.D., S.K.a.r., M.S. and S.S., N.I., F.O., I.V.C.T.D., C.M., J.O., T.S. A.M.R.K., N.S.,G.V., P.K.D., J.M. conducted experiments, and analyzed the data. G.M., H.L.N., A.R.S., S.H., W.W.Z., S.K.a.m., J.G.V., R.D., S.G., designed experiments, analyzed data and wrote the manuscript. P.L. analyzed genome data and helped write the manuscript. N.I., A.S., V.S., F.O., I.V.C., T.S., C.M., A.M., R.N., N.S., and G.V. reviewed the manuscript.

\section{Competing interests}

The FDA is currently a co-owner of two US patents that claim attenuated Leishmania species with the Centrin gene deletion (US7,887,812 and US 8,877,213). All other authors declare they have no competing interests.

\section{Additional information}

Supplementary information is available for this paper at https://doi.org/10.1038/s41467 020-17154-z.

Correspondence and requests for materials should be addressed to A.R.S., G.M. or H.L.N.

Peer review information Nature Communications thanks Nicolas Fasel and the other, anonymous, reviewer(s) for their contribution to the peer review of this work.

Reprints and permission information is available at http://www.nature.com/reprints

Publisher's note Springer Nature remains neutral with regard to jurisdictional claims in published maps and institutional affiliations.

Open Access This article is licensed under a Creative Commons Attribution 4.0 International License, which permits use, sharing, adaptation, distribution and reproduction in any medium or format, as long as you give appropriate credit to the original author(s) and the source, provide a link to the Creative Commons license, and indicate if changes were made. The images or other third party material in this article are included in the article's Creative Commons license, unless indicated otherwise in a credit line to the material. If material is not included in the article's Creative Commons license and your intended use is not permitted by statutory regulation or exceeds the permitted use, you will need to obtain permission directly from the copyright holder. To view a copy of this license, visit http://creativecommons.org/ licenses/by/4.0/

This is a U.S. government work and not under copyright protection in the U.S.; foreign copyright protection may apply 2020 